\title{
A Simple Two-Dimensional Model System to Study Electrostatic-Self-Assembly
}

\section{Citation}

Cademartiri, Rebecca, Claudiu A. Stan, Vivian M. Tran, Evan Wu, Liam Friar, Daryl Vulis, Logan W. Clark, Simon Tricard, and George M. Whitesides. 2012. A Simple Two-Dimensional Model System to Study Electrostatic-Self-Assembly. Soft Matter 8, no. 38: 9771-9791.

\section{Published Version}

doi:10.1039/c2sm26192h

\section{Permanent link}

http://nrs.harvard.edu/urn-3:HUL.InstRepos:11931825

\section{Terms of Use}

This article was downloaded from Harvard University's DASH repository, and is made available under the terms and conditions applicable to Open Access Policy Articles, as set forth at http:// nrs.harvard.edu/urn-3:HUL.InstRepos:dash.current.terms-of-use\#OAP

\section{Share Your Story}

The Harvard community has made this article openly available.

Please share how this access benefits you. Submit a story.

Accessibility 


\title{
A Simple Two-Dimensional Model System to Study Electrostatic- Self-Assembly
}

\author{
Rebecca Cademartiri, Claudiu A. Stan, Vivian M. Tran, Evan Wu, Liam Frair, Daryl
}

Vulis, Logan W. Clark, Simon Tricard and George M. Whitesides*

Department of Chemistry and Chemical Biology, Harvard University, 12 Oxford St., Cambridge,

$$
\text { Massachusetts 02138, USA }
$$

*Corresponding Author

Telephone Number: (617) 495-9430

Fax Number: (617) 495-9857

Email Address: gwhitesides@gmwgroup.harvard.edu

Journal: Soft Matter 


\section{Abstract}

This paper surveys the variables controlling the lattice structure and charge in macroscopic Coulombic crystals made from electrically charged, millimeter-sized polymer objects (spheres, cubes, and cylinders). Mechanical agitation of these objects inside planar, bounded containers caused them to charge electrically through contact electrification, and to selfassemble. The processes of electrification and self-assembly, and the characteristics of the assemblies, depended on the type of motion used for agitation, on the type of materials used for the objects and the dish, on the size and shape of the objects and the dish, and on the number of objects. Each of the three different materials in the system (of the dish and of the two types of spheres) influenced the electrification. Three classes of structures formed by self-assembly, depending on the experimental conditions: two-dimensional lattices, onedimensional chains, and zero-dimensional 'rosettes'. The lattices were characterized by their structure (disordered, square, rhombic, or hexagonal) and by the electrical charges of individual objects; the whole lattices were approximately electrically neutral. The lattices observed in this study were qualitatively different from ionic crystals; the charge of objects had practically continuous values which changed during agitation and self-assembly, and depended on experimental conditions which included the lattice structure itself. The relationship between charge and structure led to the coexistence of regions with different lattice structures within the same assembly, and to transformations between different lattice structures during agitation. 


\section{Introduction}

Crystallization and glass formation are the two microscopic processes that generate solids from liquids and gases. Understanding and controlling the formation of crystalline matter is a process of great theoretical and technological importance, and a considerable amount of work has been invested in the study of crystallization. ${ }^{1-4}$ Beyond its practical importance, crystallization is a fascinating phenomenon that occurs in many systems which are not common materials, such as stacked macroscopic spheres,${ }^{5-6}$ bubbles on a surface of a liquid, ${ }^{7}$ electrons in plasmas, ${ }^{8-9}$ and vortices in quantum fluids. ${ }^{10}$

The study of these exotic cases of crystallization is in part motivated by the hopes of drawing analogies with, and of generating insights about, the crystallization of materials. Among these model systems for crystallization, the most useful in generating new insights are probably systems of colloidal particles, ${ }^{11-18}$ which are made of particles large enough to be observed individually, yet small enough to create systems with statistically large numbers of particles.

This paper reports the development of a model system for studying self-assembly processes important in crystallization, and in the formation of forms of condensed matter in which charge-charge interactions dominate the interactions between the particles. We wished to develop a system — even if much simplified — in which we could observe the motions of all particles, and control the properties of those particles and of the environment they experienced. Our system is composed of oppositely charged (but not necessarily with the same absolute magnitude of charge), millimeter-scale polymeric spheres, agitated mechanically on a planar surface; our experiments explored the two-dimensional selforganization of these spheres. It does not replicate many of the important features of 
crystallization in ionic or molecular crystals (especially solvation, and the roles of solvent), but it has the advantages that all of the processes involved can be examined as they occur, and that most of the interactions are (or can be) known and, to a large extent, controlled. The charges of the spheres are neither quantized, nor constant during self-assembly; this characteristic limits the possibility of modeling ionic crystallization with our system but it offers the possibility of studying types of electrostatic self-assembly that are impossible in systems with fixed electrical charges. This work is part of a research program on simple electrostatic models of crystallization and polymer folding. ${ }^{19-23}$

\section{Background}

\subsection{Crystallization and self-organization}

Crystallization is one of the most fundamental processes in self-assembly, but many of its aspects are still incompletely understood. ${ }^{1-2,4}$ For example, the thermodynamics of crystal formation from solution can be measured accurately, but the interpretation of enthalpic and entropic terms is often non-intuitive, even for the simplest systems, and understanding the role of solvation of both ions and neutral molecules remains remarkably difficult. ${ }^{24}$ Even for the most elementary systems such as simple Coulombic crystals, non-equilibrium processes - nucleation, growth and dissolution of "seed" and small crystals; the formation and annealing of defects; and solvation — can be dauntingly difficult to observe and understand. ${ }^{25-29}$ 
Formation of an ionic crystal from solution—especially from aqueous solution, where both enthalpy and entropy of hydration of ions can be large and partially compensating — is almost always pictured in terms of the observable thermodynamic properties of ensembles. With the exception of occasional direct observations of individual ions on the faces of growing crystals obtained using Atomic Force Microscopy (AFM) ${ }^{28,30-33}$ it is experimentally impractical to follow the motions of individual ions equilibrating between a crystal and solution, and within a crystal. As a result, although many features of the whole ensemble can be measured, processes that involve single or small numbers of ions are usually inferred indirectly. In studies of systems of crystallizing colloids, ${ }^{11-14}$ more information can be extracted about individual objects, but again these systems are often too complicated to follow all relevant particles, and events involving small particle numbers usually are not detectable.

\subsection{Tribocharging: Materials and mechanisms}

The charge of molecular ions is quantized, and changes in steps corresponding to the gain or loss of one electron. An alternative class of electrostatic systems for the study of crystallization is that of meso- or macro-scale objects that are electrostatically charged. An attractive class of such materials is those that are tribocharged: that is, charged as the result of contact with another material. Tribocharging is one of the oldest subjects in physical sciences. Around 600 B.C. Thales of Miletus charged fur and amber by rubbing them together, and investigations of tribocharging have continued idiosyncratically ever since, but tribocharging as a stand-alone subject in materials science has (re)emerged only recently. 
When two materials are put in contact, one tends to charge positive and the other negative; this phenomenon allows the ranking of materials, according to their tendency to charge positively or negatively, into a triboelectric series—an empirical concept introduced first in 1757 by Johan Carl Wilcke. ${ }^{34}$ The triboelectric series remains an actively investigated topi $^{35}$ because it is very useful but not fully accurate - the ranking of materials depends on contributions from several different processes and on environmental conditions such as ambient humidity, and many exceptions from the charging rules of the triboelectric series, such as the existence of cyclic triboelectric series, ${ }^{36}$ are known.

The most important goal of modern research in triboelectricity is the understanding the microscopic mechanisms that cause tribocharging. Here we will only present these mechanisms and point the reader to reviews ${ }^{37-39}$ that discuss these mechanisms in detail. At least four major mechanisms appear to cause tribocharging. (i) Electron transfer occurs when dissimilar metals or semiconductor come in contact; the hypothesis of tribocharging by electron transfer produces good predictions of the tribocharging of metals, ${ }^{37}$ but attempts to extend its applicability to insulators ${ }^{39}$ have been challenged on theoretical ${ }^{38}$ and experimental ${ }^{40}$ bases. (ii) Ion transfer dominates tribocharging in species having both mobile and fixed ions; ${ }^{38}$ the validity of this mechanism has been strongly supported by the ability to synthesize materials that have predesigned tribocharging properties. ${ }^{38,41-42}$ (iii) Material transfer may be important in some systems, such as metals sliding under dry wear conditions ${ }^{43}$ or polymer-polymer contacts. ${ }^{44}$ (iv) Differential adsorption of hydroxide and hydronium ions from a thin film of water formed at contact sites ${ }^{38}$ can explain the correlation between tribocharging and electrochemical material properties such as the zeta function, and applies to both ionic and non-ionic insulators; the discovery that tribocharging still occurs, 
but is less effective, in the absence of water, ${ }^{44}$ indicates that this mechanism might only contribute, rather than dominate, tribocharging.

The relative rates and contributions of these charging processes, and the rates of discharging to air or other materials, lead to a steady-state surface charge density if the tribocharging is continued indefinitely. Charge dissipation processes ${ }^{45-46}$ are therefore an integral part of triboelectrification, which would otherwise continue until the charges build up to much larger values than those observed experimentally. The extent to which materials would charge in the absence of charge relaxation is not known, but a lower limit is suggested by the emission of bursts of X-rays when adhesive tape is unwound under vacuum - $\mathrm{a}$ phenomenon caused by the tribocharging of the adhesive tape.$^{47}$ The intensity of these X-rays can be explained by the transient ( $\sim \mathrm{ns})$ existence of surface charge densities approximately one hundred times larger than the largest static surface charge densities observed in triboelectrification.

Another direction of research on tribocharging concerns exceptions from the triboelectric series model; one exception that received significant interest recently is the phenomenon of tribocharging between two objects made from the same material. A few different types of physical systems exhibit this exception: (i) if the objects have different sizes, the smaller one acquires a negative charge, and charge separation appears to be caused by a size-dependent capacity of the objects to provide and accept charge carriers; ${ }^{39}$ (ii) identical particles can acquire different charges if they are electrically polarizable and collide in the presence of an external electric field; ${ }^{48}$ and (iii) identically shaped objects made from the same nonelemental material can tribocharge due to random microscopic fluctuations in the composition of the surface. ${ }^{49}$ 


\section{Experimental design}

\subsection{Tribocharged objects as a model system}

We use tribocharging of polymeric objects (most commonly, spheres made of materials chosen for their position in the triboelectric series), as the basis for this work. The system is straightforward in concept: we place two (or sometimes three) different types (in composition, size, shape, and number) of objects on a flat surface (typically made from aluminum) and agitate it. The whole system (dish and objects) is electrically insulated and neutral. The individual objects charge, and self-organize according to the interaction of two processes - electrostatic (most importantly, attractive and repulsive forces between the charges on the objects, but also interactions with the dish), and mechanical (collisions with other objects and with the walls of the dish; friction between objects, and between objects and the dish). The magnitudes of the charges depend on the details and history of the system, but their steady-state values are roughly constant. Many aspects of the system are thus under our control. An important advantage of this system is that we can observe and track (if we need to) all the objects in an experiment.

\subsection{Design and operation}

The experimental apparatus is based on a design that we reported previously. ${ }^{20}$ The main considerations in the design of the apparatus were to be able switch between different 
configurations quickly and easily (i.e., in less than a minute), to record the position of the objects continuously, to measure the charge on the objects easily, and to ensure reproducibility.

The apparatus consists of a level surface supported from the side, below or above, depending on the motion actuator that we used (see Fig. S1(a) in the ESI $\dagger$ ). The actuator shakes the level surface. Orbital, linear, or a combination of both types of actuators produced shaking motions that were orbital, linear, or randomly planar, respectively. We attached an open flat dish with perpendicular walls to the shaking surface using adhesive tape and polyurethane foam in order to insulate electrically the system from its environment, exchange the dish easily, and maintain a reproducible alignment; we then filled the dish with polymeric objects (spheres, cylinders, cubes) in a random arrangement (Fig. S1(b)-(c), ESI†). We placed a video camera vertically above the dish to record the movement of the objects continuously, and placed a Faraday box for the measurement of the charge of the objects next to the shaking surface. The whole system was situated in a humidity-controlled environment (glove bag).

Prior to each experiment, the polymeric objects in the dish were rinsed with ethanol, dried with a stream of nitrogen gas, and discharged (with an antistatic gun; Zerostat 3, Sigma-Aldrich Corp.) to achieve the same starting conditions in all experiments. We placed the objects in the dish and started the agitation; the objects moved over the surface of the dish during agitation and collided with its walls and other objects, tribocharging during movement and collisions. The resulting electrostatic forces led to the self-assembly of the objects into structures that were either disordered or ordered (cubic, rhombic, or hexagonal) depending on materials, history, and environment. We determined the degree of order of the structures and 
the type of lattice structure through image analysis of the movies recorded with the camcorder (see the ESI $\uparrow$ for movie examples). After the end of agitation, we measured the electrical charges of the spheres using an electrometer connected to the Faraday box.

\subsection{Fixed experimental conditions}

We chose to keep the humidity of the air and the surface coverage of the dish (i.e. the ratio of area covered by objects to the total area of the dish) constant in all experiments. The humidity can influence the charge accumulation on the objects ${ }^{37}$ and thus the process of selfassembly. Grzybowski et al. ${ }^{20}$ reported that changes in humidity between $\mathrm{RH}=8 \%$ and $50 \%$ had no influence on the self-assembly of spheres. We kept a low $(\mathrm{RH}=4 \% \pm 2 \%)$ relative humidity by purging the enclosure of the system (a glove bag) with nitrogen gas; this low values reduced the rate of dissipation of the charge, and increased the reproducibility in the experiments.

The total number of spheres used in experiments (for a dish of a given size) influenced the self-assembly. We normalized the number of spheres for dishes of different sizes and shapes using the surface coverage parameter, which we defined as the ratio between the projected area of all spheres and the area of the dish. A surface coverage of $33 \%$ of the dish led to the formation of a rich variety of structures in preliminary experiments, and made it attractive as a baseline experiment with which to study the influence of other variables. A lower surface coverage led to fragmented structures distributed over the whole dish; structures assembled at a higher surface coverage were primarily dependent on the initial arrangement of objects before agitation, and not on the electrostatic forces generated during 
agitation. We have described previously ${ }^{20}$ similar structures for Nylon and Teflon spheres at surface coverages around $35 \%$.

\subsection{Variable experimental conditions}

Our simple setup allowed us to change experimental conditions easily. The variables that we changed during the experiments were the kinematics of the shaking motion, the frequency of agitation in the case of the linear shaking, the material of the objects and dish, the size and shape of the objects and dish, and the "concentration" (i.e. the number fraction in the mixture) of the objects.

3.4.1. Type of shaking motion and agitation frequency. We employed three different modes of shaking - orbital, linear, and pseudo-random ${ }^{23}$ (a combination of orbital and linear motions) - to observe how the movement of the objects influences their self-assembly (Fig. S1(a) in the ESI $\dagger$ ). For the linear shaking we varied its frequency at constant amplitude to study the self-assembly of spheres at different agitation energies.

3.4.2. Material of the objects and dish. Our objects and dishes were made from materials located at different points within the triboelectric series (Table ST1 in the ESI $\dagger$ ). We expected materials from the top of the triboelectric series (e.g., Nylon, Delrin) to charge strongly positively (i.e., absolute charge density $\sigma>25 \mathrm{pC} / \mathrm{mm}^{2}$ for spheres), and materials from the bottom of the series (e.g., polystyrene, Teflon, Torlon) to charge strongly negatively (i.e., $\sigma<-25 \mathrm{pC} / \mathrm{mm}^{2}$ for spheres). We expected materials from the middle of the triboelectric series (e.g., aluminum, poly(methyl methacrylate), polypropylene, high density polyethylene) to charge weakly, either positively or negatively (i.e., $\sigma<9 \mathrm{pC} / \mathrm{mm}^{2}$ for spheres). In general, 
the magnitude of charge on the objects is expected to be higher the further apart the materials are in the triboelectric series,${ }^{35}$ up to a limit set by whatever types of discharge processes (e.g., discharge to air) operate; our preliminary experiments confirmed these expectations. We chose Nylon and Teflon spheres for most of our experiments because they charged strongly. Another combination of spheres that acquired high charges was Delrin and Torlon; they were our second choice of materials.

3.4.3. Shape and size of the objects and dish. The physical size of the system (millimeters to centimeters) provided us with many options in designing individual experiments, because the components of the system can be formed into different shapes and sizes using standard machining.

3.4.3.1. Shape of the objects. We employed spheres in most experiments (Fig. S1(c) in the ESI $\dagger$ ), because their shape (i) resembles that of ions, (ii) allows them to roll (rather than slide) over the support, and (iii) is compatible with the simplifying assumption that the charge accumulated on the surface is homogeneously distributed. We used cubes and cylinders (Fig. S1(c) in the ESI $†$ ) in a few experiments because their charging and assembly was qualitatively different from spheres. While spheres tribocharged by contact and separation over their entire surface, cubes tribocharged on their lower surface by friction, and on their sides by contacts and sliding against other objects. Another difference between spheres and cubes is that the shape of the cubes makes it impossible for them to form closepacked hexagonal lattices. Cylinders were qualitatively different from both spheres and cubes because of their anisotropy—cylinders can have aspect ratios (length/diameter) larger than one, and they can roll without sliding only in the direction perpendicular to their centerline. 
3.4.3.2. Shape and size of the dish. The dishes had circular, triangular, and square shapes (Fig. S1(b) in the ESI†). The corners of triangular and square dishes allowed us to study the effect of confinement on the lattice structure of spheres. Circular dishes cannot confine spheres at the corners, but can influence the lattice structure due to the curvature of their walls.

3.4.3.3. The absolute and relative size of the objects. We combined two types of spheres with different diameters—-from $\mathrm{d}=2.38 \mathrm{~mm}$ to $\mathrm{d}=6.35 \mathrm{~mm}$ - to simulate lattices formed from ions of various sizes (e.g., $\mathrm{Na}^{+} \mathrm{Cl}^{-}$vs. $\mathrm{Cs}^{+} \mathrm{Cl}^{-}$, Fig. S1(c) in the $\left.\mathrm{ESI} \dagger\right)$. We varied the diameter of circular dishes (from $\mathrm{d}=40 \mathrm{~mm}$ to $\mathrm{d}=140 \mathrm{~mm}$ ) while keeping the diameter of the spheres the same $(d=3.18 \mathrm{~mm})$. This change in the diameter of the dishes affected the assembly in two ways. First, it changed the ratio of the radius of curvature of the walls to the spacing of the lattice; as this ratio became smaller, the lattices had to distort more from their

ideal structure to match the shape of the wall. Second, it changed the total number of spheres on the dish and the relative number of spheres within assemblies that contacted the walls during shaking; a larger fraction of the spheres is affected by interactions with the wall in smaller dishes, and "confinement effects" become more important.

3.4.4. The relative proportion of the objects. We combined Nylon $(\mathrm{N})$ and Teflon $(\mathrm{T})$ spheres at various number fractions $\chi_{N}\left(\chi_{N}=n_{N} /\left(n_{N}+n_{T}\right)\right)$ to model lattice structures with the composition formula $\mathrm{A}_{\mathrm{x}}{ }^{+} \mathrm{B}_{\mathrm{y}}{ }^{-}$and $\mathrm{x} \neq \mathrm{y}$.

\subsection{Analysis of data}


3.5.1. Image analysis. The millimeter-scale of the objects, and their low velocities (less than $1 \mathrm{~m} / \mathrm{s}$ ) allowed us to measure their positions accurately using a video camera; a frame every second was usually sufficient to follow the evolution of self-assembly. Our design, if augmented with a high-speed video camera, would allow the determination of both the positions and velocities of all objects; a consumer-grade video camera was sufficient for this study. We analyzed the movies using a set of MatLab programs that quantified the degree and type of short- and long-range order in the assemblies. The program first determined the coordinates of each sphere in a frame of the movie and then processed this information to evaluate the spatial organization of assemblies.

\subsubsection{Determination of short-range order using the distribution of angles between} neighbors $(A B N)$. We analyzed the spatial distribution of neighboring spheres of different types to evaluate (i) the degree of close-packing within assemblies and (ii) the fraction of spheres that were part of ordered close-packed structures. Our experiments led to four types of ordered close-packed lattices (Fig. S2(a) in the ESI†). Mixtures that were 1:1 formed square (S), rhombic (R), and layered-hexagonal lattices (HL: a hexagonal close-packed structure in which the two types of spheres occupy alternating rows); 2:1 mixtures formed distributed hexagonal lattices (HD: hexagonal close-packed structure formed of units of one rarer type of sphere surrounded by six of the other type).

We quantified the spatial distribution of neighboring spheres by measuring the distribution of angles between lines defined by spheres of different types (we will therefore refer to this distribution using the acronym $\mathrm{ABN}$ or "angle between neighbors"). The calculation has two stages; first, for each sphere, all these angles are calculated; second, the angles for all spheres of a given type are collected. The ABN is the distribution of all these 
angles. When the $\mathrm{ABN}$ of ordered assemblies is plotted as a histogram, it has peaks that are characteristic of the type of lattice structure: $90^{\circ}$ angles are characteristic of square lattices, and $60^{\circ}$ and $120^{\circ}$ angles are characteristic of hexagonal lattices (Fig. S2(b) in the ESI $\dagger$ ).

\subsubsection{Determination of the fractions of spheres in a given type of lattice from the $A B N$}

distribution. We used the peaks in the $\mathrm{ABN}$ to calculate the number of spheres in a given assembly that were part of each type of lattice or in disordered assemblies. For example, in a perfect infinite square lattice the number of spheres is equal to the number of $90^{\circ}$ angles divided by four: each sphere is surrounded by four neighbors of opposite type separated by $90^{\circ}$ angles; the image analysis procedure therefore finds four $90^{\circ}$ angles for each sphere. In a first approximation, (i) the number of spheres in a square lattice is equal to the number of $90^{\circ}$ angles divided by four, and (ii) the fraction of spheres in a square lattice is this number divided by the total number of spheres. Equations $\mathrm{SE}(1)$ to $\mathrm{SE}(4)$ in the supplementary information (see ESI $\dagger$ for details) give the formulas that we used to calculate the numbers of spheres in S, HL, and HD lattices.

Equations $\mathrm{SE}(1)$ to $\mathrm{SE}(4)$ are quantitatively accurate only in the case of perfect lattices made from a very large number of spheres. When they are applied to finite-sized crystallites they underestimate significantly the lattice fractions because they do not account for the case of spheres at edges and grain boundaries; for example, equation SE(1) applied to a perfect four-by-four square lattice gives a square lattice fraction of only $56 \%$. The lattice fractions that we measured were lowered further by the incomplete detection of $\mathrm{ABN}$ angles due to poor image contrast in some of the movies. Despite these shortcomings, we chose to use a relatively simple $\mathrm{ABN}$ analysis because it was reproducible and produced qualitatively accurate trends for a given series of experiments (e.g., varying the frequency of agitation). 
As a guide for interpreting the lattice fractions that we computed, we note that (i) for disordered assemblies (as judged by visual inspection) all ABN lattice fractions were below approximately 0.05 , (ii) $\mathrm{ABN}$ lattice fractions above 0.1 corresponded to visible order of that type in the assembly, and (iii) ABN lattice fractions above 0.2 corresponded to assemblies in which most of the spheres were in one type of lattice but the assembly contained multiple crystallites. Further details of the ABN-based processing can be found in the supplementary information (see ESI† for details).

\subsubsection{Determination of the long-range order from the distance between spheres (DBS).} We used the coordinates to measure the distances between every pair of spheres of different type, and we calculated the histogram of these distances; we will therefore refer to the distribution of distances between spheres of opposite type and to its histogram as DBS. The DBS of assemblies with crystalline order exhibited peaks at various distances. The DBS peaks were characteristic of a given lattice structure. We used the height of the peaks to perform qualitative comparisons between samples (e.g., the higher the peak, the greater the probability of finding two spheres separated by that distance, Fig. S2(c) in the ESI†े). Narrow peaks indicated that a given type of lattice symmetry was prevalent in the assembly, and distinguishable peaks located at large distances indicated that crystalline order was preserved over extended regions. Further details of the DBS-based processing can be found in the supplementary information (see ESI $\uparrow$ for details).

3.5.2. Determination of the electrical charge of the objects. We found that the charges on different objects of one type from the same crystalline domain were not exactly the same, but the average values of the charge on a given type of object were reproducible. We have therefore measured at least ten objects of each type in every region that had a defined lattice 
structure, for every self-assembly experiment, to determine the average charge of the objects. To measure the charge we moved the objects one by one, using polybutylene terephthalate (PBT) tweezers, into a Faraday box connected to an electrometer which then measured the charge of the sphere.

The choice of tweezers used was dictated by the requirement that handling the charged objects should have a negligible effect on the measurement of charge. We have shown previously that PBT tweezers preserve the charge of the objects better than stainless steel or wood-tipped tweezers, ${ }^{22}$ and we determined recently ${ }^{50}$ that the charge of the objects changes by at most $2-4 \%$ after multiple contacts between the objects and the PBT tweezers.

We performed the measurements of charge shortly after the end of agitation - typically within 5-10 minutes - to minimize any possible leakage of charge from the objects. To estimate the extent and timescale over which charge leaked from the objects, we monitored the charge of Teflon and Nylon spheres mixed in an aluminum dish over a period of 48 hours after agitation (Fig. S4 in the ESI†). The charge of Teflon spheres remained constant, and that of Nylon spheres decreased by $20-25 \%$ after the first four hours and then remained constant. Assuming that the kinetics of the leakage of charge was exponential, we evaluated that Nylon spheres would lose less than $5 \%$ of their charge during the time required for measurements.

\subsection{Reproducibility tests}

We tested reproducibility by repeating all the experiments under the same set of conditions at least seven times, and analyzing the structure and charge for each trial individually. Two sets 
Fig. 1 Study of reproducibility for seven samples of Nylon (dark) and Teflon (light) spheres on aluminum dishes in a 7:3 number ratio, assembled under the same conditions. a) Photographs of steady-state structures. b) DBS distributions at 180 seconds. Slight shifts in the location of the peaks were caused by the finite resolution of the binning. c) Average charge of 10 Nylon (positive) and 10 Teflon (negative) spheres after 180 seconds. The error bars represent one standard deviation. 

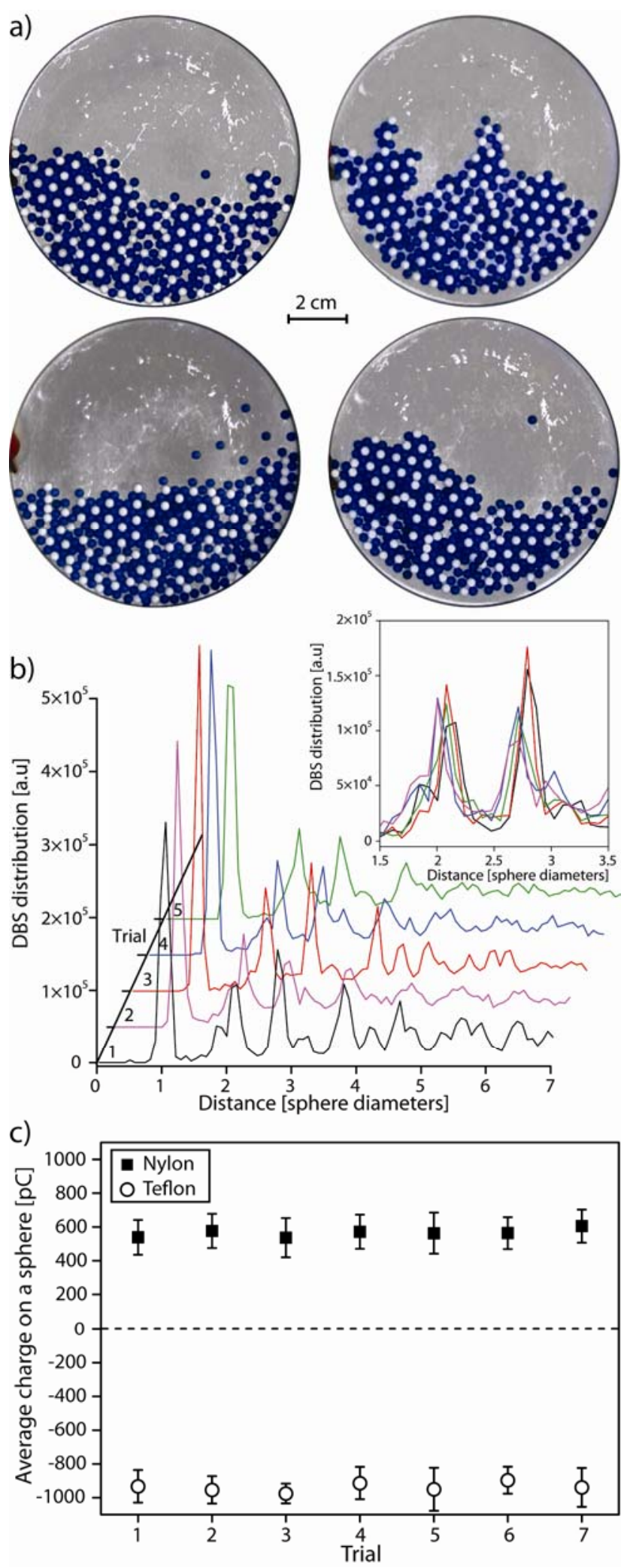
of spheres and dishes were used for every data point. Fig. 1 presents the results obtained for an experiment with a 7:3 number ratio of Nylon:Teflon spheres on aluminum dishes. The structures found in these trials were qualitatively similar to one another, but not identical (Fig. 1(a)). If crystalline order developed, the type of lattice was the same (Fig. 1(b)). The assemblies were composed of several crystalline domains whose sizes and orientations varied from experiment to experiment. The average charge on one type of sphere was practically constant in all of the experiments - the experiment-to-experiment variation of charge was less than the standard deviation of charge measurements (Fig. 1(c)).

\section{Materials and methods}

\subsection{Materials}

Nylon-6,6 (Nylon), polytetrafluoroethylene (Teflon), polyamide-imide (Torlon), high density polyethylene (HDPE), polyoxymethylene (Delrin), polypropylene (PP) and polystyrene (PS) spheres with various diameters $(2.38 \mathrm{~mm}$ to $6.35 \mathrm{~mm})$ were purchased from McMaster Carr Supply Company (USA).

Dishes with various shapes and sizes and smooth surfaces were machined from aluminum, Nylon, Delrin, Teflon, and poly(methyl methacrylate) (PMMA). Their rims were colored using white out or black paint to provide better contrast for analytical purposes. Technical ethanol (95\% concentration) was bought from Sigma-Aldrich Corp. 


\subsection{Staining the polymer objects}

Because all polymers that we tested, except for Torlon, were clear or white, we had to dye one of the types of polymer objects to identify them visually; we dyed all types of polymer objects except Torlon and Teflon. We measured the effect of dyeing on the tribocharging of Nylon spheres agitated with PS spheres on a gold surface (see Fig. S3 in the ESI $\dagger$ ). Dyed Nylon spheres acquired approximately 5-10\% less charge than not-dyed Nylon spheres, a difference which was comparable but less than the standard deviation of charge on the spheres; dyeing influences tribocharging, but does not influence the self-assembly qualitatively.

We dyed the objects by immersing them in an aqueous dye solution (e.g., Disperse Blue 14, Disperse Red 1, and Disperse Black 9 from Sigma-Aldrich Corp.) at a temperature of 100 ${ }^{\circ} \mathrm{C}$ for $60 \mathrm{~min}$. Dyed objects were rinsed with water and $95 \%$ ethanol and dried overnight at $60^{\circ} \mathrm{C}$. Torlon was used as purchased, and Teflon could not be dyed by this method.

\subsection{Experimental apparatus}

In order to create a humidity-controlled environment, we performed the experiments in a glove bag (ERLAB Inc. USA); $\mathrm{N}_{2}$ flowed into the enclosure and the relative humidity inside the enclosure was measured with a hygrometer (VWR International). The rate of flow of nitrogen was adjusted to maintain humidity at $4 \% \pm 2 \%$ at room temperature $\left(24{ }^{\circ} \mathrm{C} \pm 2{ }^{\circ} \mathrm{C}\right)$. 
A high definition camcorder (Canon VIXIA HD20) was used to record movies during agitation.

We used linear motors from LinMot, Inc. (P01-23x80) and orbital shakers from IKA Works, Inc. (MS3 Digital) and Madell Technology Corp. (ZD-9556-A). Typical agitation frequencies and amplitudes were: $v_{\mathrm{L}}=8 \mathrm{~Hz}, \mathrm{~A}_{\mathrm{L}}=9 \mathrm{~mm}$ for linear agitation, and $v_{\mathrm{O}}=750$ $\mathrm{rpm}, \mathrm{R}_{\mathrm{O}}=2.25 \mathrm{~mm}$ for the orbital agitation; and $v_{\mathrm{O}}=130 \mathrm{rpm}, \mathrm{R}_{\mathrm{O}}=5.1 \mathrm{~mm}, \mathrm{~A}_{\mathrm{L}}=8 \mathrm{~mm}, v_{\mathrm{L}}$ $=3.6 \mathrm{~Hz}$ for pseudo-random agitation.

\subsection{The measurement of electrical charge}

4.4.1. Measurement of charge on the objects. We measured the charge on the polymer spheres by picking up each sphere with electrically insulating and non-charging (i.e. antistatic) PBT tweezers, and dropping the spheres into a Faraday box. An electrometer (Keithley 6514) measured the charge inside the box, and a homemade LabView program recorded the charge as a function of time. The charge on a sphere was equal to the difference in the charge measured by the electrometer before and after the sphere entered the box.

4.4.2. Measurement of the charge of the system during agitation. Since the system was electrically insulated and was discharged before agitation, we expected the system to remain approximately electrically neutral during agitation. We monitored the electrical charge of the whole system during agitation by connecting the aluminum dish directly to the electrometer (see Fig. S4 in the ESI $\dagger$ ).

\subsection{Computer analysis of the movies}


The movies recorded by the video camera were processed one frame at a time. For each frame, we used a home-built MatLab program that processes the image to find the position and the type of every object in the dish. For certain combinations of dish and object materials, the low contrast of some images prevented all objects from being detected by the program; we used the criteria that at least $90 \%$ of the objects must be detected to perform the next steps of image analysis. Using the position of each sphere, the program computed the $\mathrm{ABN}$ and DBS distributions (as described in Experimental design and in the supplementary information: see ESI $\uparrow$ for details); the properties of self-assembled structures were obtained by averaging $\mathrm{ABN}$ and $\mathrm{DBS}$ distributions for multiple movies.

\section{Results}

We observed self-organization phenomena-which we define as reproducible changes in the spatial organization of the objects due to mechanical agitation of the dish — under many experimental conditions. The number of experimental parameters was large: the shape, size, and material of the dish and of the objects (nine parameters), the type, amplitude and frequency of the shaking motion (three to five parameters), and the number of spheres of each type (two parameters). A complete investigation of the effect of all these parameters and of the relationships between these effects was impractical. We have therefore surveyed these effects, and we chose to investigate in detail only the effect of those parameters and combinations of parameters (such as the ratio of the numbers of different types of objects) 
that we expected to provide significant information about self-assembly; we kept the rest of parameters at fixed values that provided the best conditions for self-assembly.

We used two observables to characterize the assemblies: the structure of the lattice, and the electrical charge of the objects. The characterization of assemblies by their lattice structure is a usual procedure in the fields of crystallization and self-organization. The characterization of the assemblies by the charge of their components is specific to our system; in microscopic electrostatic crystals the charge of ions remains constant during selfassembly. In our system the charge of an object, which was generated by tribocharging, depended on its environment and on the history and details of the experiment. In a first approximation, the environmental conditions that influence tribocharging are the rate and the types of contact-and-separation events that an object experiences (e.g., between object of type A and object of type A, between object of type A and an object of type B, between an object of type A and the dish). Measuring the charge of the objects helped us understand why one lattice structure formed preferably over another.

We expected that the overall system (all objects and the dish) was charge neutral, because the system was electrically isolated; the only ways in which the charge of the system could change was by leakage of charge through the plate that supported the dish or though the atmosphere. To demonstrate that such leakage was negligible, we agitated Nylon and Teflon spheres $\left(\mathrm{n}_{\text {total }}=340\right)$ in different number ratios on circular aluminum dishes $(\mathrm{d}=100 \mathrm{~mm})$ and measured the charge of the whole system by connecting the dish with the spheres to an electrometer. The average total charge (spheres plus dish) measured by the electrometer increased slowly, and after three minutes of agitation it was between 500 and $3000 \mathrm{pC}$, a value which was less than the average charge on five spheres in a system composed of 340 
spheres. We monitored the charge of the whole system in several other experiments (see Fig. S4 in the ESI†); the charge was always less than $3 \%$, and usually less than $1 \%$, of the net charge of all spheres. The small charge of the whole system justified our approximation that the whole system could be considered to be electrically neutral.

\subsection{The best experimental conditions for the study of self-assembly}

5.1.1. The effect of the type of agitation. Agitation was necessary to bring the objects into contact, and thus to enable the tribocharging that led to self-organization. The tribocharging of spheres occurred by collisions, rolling and sliding between the spheres and the spheres and dish; collisions and rolling were the dominant charging mechanisms. We compared linear agitation, orbital agitation and a combination ${ }^{23}$ of orbital agitation with randomly timed 'kicks' (which we will call thereafter "pseudo-random agitation") in their ability to tribocharge objects and to form ordered structures.

We used Nylon and Teflon spheres $\left(\mathrm{n}_{\mathrm{N}}=\mathrm{n}_{\mathrm{T}}=170, \mathrm{~d}_{\mathrm{N}}=\mathrm{d}_{\mathrm{T}}=3.18 \mathrm{~mm}\right)$ in a circular aluminum dish $(\mathrm{d}=100 \mathrm{~mm})$ to study the different types of agitation (Fig. 2). The frequencies and amplitudes of the shakers were optimized to induce the most ordered structures in a reasonable time (three minutes) under the constraint that all spheres remained in contact with the dish. If objects lost contact with the surface of the dish (e.g., moved on the top of other spheres) the surface coverage of the dish and the tribocharging behavior of the objects changed.

The self-assembly was composed of two distinct but interacting processes: the charging of spheres, and their spatial redistribution. Depending on the experimental conditions, self- 
assembly had up to three stages: (i) initial charging and formation of small clusters (two-five spheres, including at least one of each type), (ii) further charging and assembly of these clusters into larger assemblies, and (iii) formation of assemblies with higher crystalline order through rearrangements within the large assemblies (sometimes with further changes in the charge). Under continued agitation, the system assumed a steady-state type of structure and the spheres charged to steady-state values. In experiments with different types of agitation, the Nylon and Teflon spheres always developed the same high steady-state charges $\left(\mathrm{Q}_{\mathrm{N}} \approx\right.$ $650 \pm 100 \mathrm{pC}, \mathrm{Q}_{\mathrm{T}} \approx-750 \pm 100 \mathrm{pC}$ ) (Fig. 2(b)).

The pseudo-random agitation (Fig. S5(a) in the ESI†) led to strong, abrupt movements of the spheres along the direction of the 'kicks' with a smaller rotational movement superimposed. The spheres took the longest time to assemble under this type of movement (about 70 seconds for the formation of small clusters and 100 seconds for formation of the larger assembly) and there was no significant rearrangement once the structure had formed. The steady-state assembly was compact and had a mixed structure with ordered lattices (layered hexagonal, square, and rhombic) on the periphery of the assembly and a disordered close-packed structure with occasional 'rosettes' (made from seven close-packed spheres in hexagonal symmetry) on the inside of the assembly (Fig. 2(a)). The assembly formed during pseudo-random agitation expelled approximately $15 \%$ of the Teflon spheres; these spheres spread on the surface of the dish or accumulated along its wall. The ABN of the steady-state assemblies typically had a broad peak between $55^{\circ}$ and $140^{\circ}$ (reflecting the presence of rhombic lattices and disordered areas) and two superimposed narrow peaks centered on $60^{\circ}$ and $120^{\circ}$ (characteristic of the hexagonal-layered symmetry). 
Fig. 2 Influence of the modes of agitation on the charge and structure of assemblies of mmsized spheres. a) Photographs of steady-state structures found for 1:1 mixtures of Nylon (dark) and Teflon (light) spheres on aluminum dishes. The arrows indicate the trajectory of the shaking motion for pseudo-random motion $\left(v_{\mathrm{O}}=130 \mathrm{rpm}, \mathrm{R}_{\mathrm{O}}=5.1 \mathrm{~mm}, v_{\mathrm{L}}=3.6 \mathrm{~Hz}, \mathrm{~A}_{\mathrm{L}}\right.$ $=8 \mathrm{~mm})$, orbital motion $\left(v_{\mathrm{O}}=750 \mathrm{rpm}, \mathrm{R}_{\mathrm{O}}=2.25 \mathrm{~mm}\right)$, and linear motion $\left(v_{\mathrm{L}}=7 \mathrm{~Hz}, \mathrm{~A}_{\mathrm{L}}=9\right.$ $\mathrm{mm})$. b) Average steady-state charge on the Nylon and Teflon spheres. The error bars represent one standard deviation calculated from all trials; for each trial we measured the charge of approximately 10 spheres of each type. 

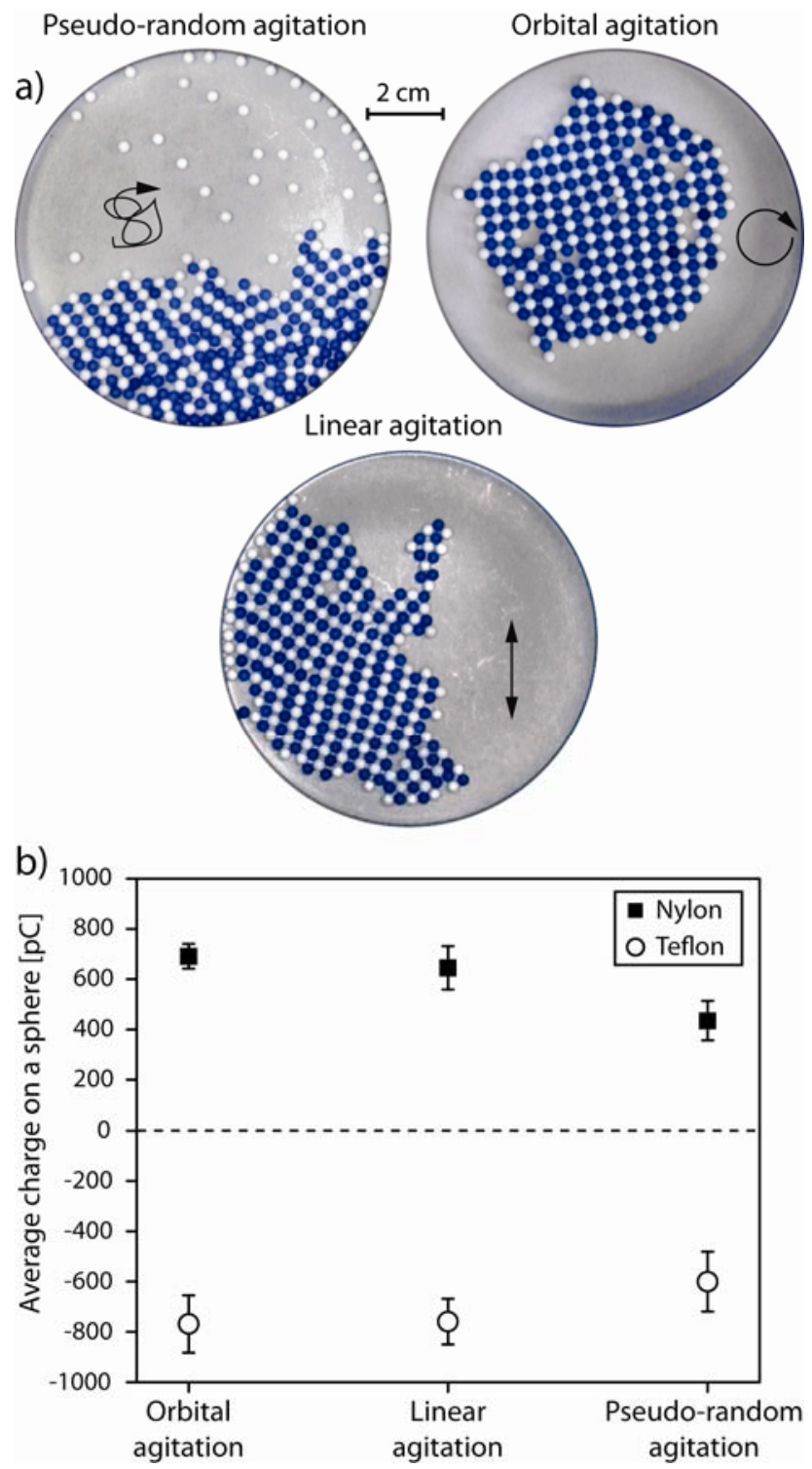
The orbital agitation (Fig. S5(b) in the ESI†) caused a faster assembly of spheres than the pseudo-random one (about 30 seconds for the formation of small clusters and 60 seconds for assembly). Single loose spheres rolled over the dish until they found a binding site in the assembly. The initial structure rearranged later by moving spheres and small crystallites inside the assemblies along their weakest interaction points (e.g., single-sphere contacts or line defects, Fig. S6(a) in the ESI $\uparrow$ ). The steady-state structure formed had a highly ordered square lattice with few point and line defects (Fig. 2(a)).

The linear agitation (Fig. S5(c) in the ESI $\dagger$ ) produced the fastest assembly (about 10 seconds for the formation of small clusters and 30 seconds for assembly). The initial assembly rearranged quickly through two mechanisms. In the first mechanism, parts of the crystal broke off, rearranged, and reattached: this process increased the degree of order of the structure (Fig. S6(b) in the ESI $\dagger$ ). This rearrangement process had a duration of approximately three seconds and repeated until the best-ordered structure formed. In the second mechanism, spheres inside crystallites changed their distance to each other and thus their lattice parameters. For example, spheres in a hexagonal-layered lattice increased their average nearest neighbor distance to the same type of sphere and transformed into a square lattice going through the intermediate stage of a rhombic lattice (Fig. S6(c) in the ESI $\dagger$ ). In general, the transition from hexagonal-layered lattices to square lattices was neither complete nor irreversible; the lattices could also remain in a rhombic symmetry, and we also observed the reverse process of square lattices transforming into hexagonal-layered lattices. The steady-state was thus characterized by a mixture of hexagonal-layered and square lattice crystallites, and the transformation from one lattice type to another took approximately three or four seconds. The two types of rearrangement processes encountered during linear 
agitation led to highly ordered steady-state structures with close to $100 \%$ square and hexagonal-layered arrangements of the spheres.

The comparison of the linear, orbital and pseudo-random agitation demonstrated that the type of agitation influenced the structure formed. Agitation that did not cause rearrangements (pseudo-random) led to partially ordered structures, while agitation that caused rearrangements (linear or orbital) led to highly ordered structures. The three types of rearrangements that occurred during linear agitation (movement of spheres, detachment and reattachment of crystallites, and changes in the lattice spacing) made linear agitation preferable to orbital agitation, where only two mechanisms of rearrangement occurred (movement of spheres, and rearrangement along grain boundaries). We decided to use linear agitation for all further experiments, because we expected that this type of agitation would provide the most efficient annealing of structures and thus lead to the most ordered assemblies.

A likely cause for the different effects of the three types of agitation is the difference in the accelerations they imparted to the dish during shaking, because higher dish accelerations transferred more momentum to the objects. We estimated that the acceleration of the dish during pseudo-random shaking had variable direction and a magnitude on the order of $1 \mathrm{~m} / \mathrm{s}^{2}$ with brief increases up to $5 \mathrm{~m} / \mathrm{s}^{2}$ after the kicks delivered by the linear actuator. The direction of acceleration of the dish during orbital shaking rotated and its magnitude was constant around $15 \mathrm{~m} / \mathrm{s}^{2}$. During linear actuation the dish experienced brief periods of strong acceleration along the direction of movement as the actuator changed its direction of motion; we estimated that the peak acceleration was on the order of $100 \mathrm{~m} / \mathrm{s}^{2}$. The three types of 
shaking thus produced significantly different dish accelerations; faster self-assembly and better ordered assemblies were correlated with higher peak accelerations.

It is instructive to compare the peak accelerations with the highest dish acceleration that the spheres can follow without sliding, which we estimated to be between 10 and $20 \mathrm{~m} / \mathrm{s}^{2}$ using the friction coefficients of different sphere materials on an aluminum dish. Spheres therefore only rolled during pseudo-random shaking, were on the threshold of sliding during orbital agitation, and briefly slid during each cycle of linear shaking. It is possible that the brief sliding motion enabled the additional rearrangement mechanisms that we observed during linear agitation.

5.1.2. The influence of the frequency of linear agitation. A higher frequency of the linear shaking increased both the frequency and the duration of the bursts of dish accelerations, and therefore led to more momentum being transferred from the dish to the spheres. In addition to identifying optimum experimental conditions, a study of the dependence of the self-assembly on the shaking frequency provided a way to investigate the dependency of self-assembly on the momentum of the spheres.

We used Nylon and Teflon spheres $\left(\mathrm{n}_{\text {total }}=340, \mathrm{~d}=3.18 \mathrm{~mm}\right)$ in a 1:1 mixture on a circular aluminum dish $(\mathrm{d}=100 \mathrm{~mm})$ and we varied the frequency of the linear actuator from 3 to $12 \mathrm{~Hz}$ while keeping the amplitude $(\mathrm{A}=9 \mathrm{~mm})$ and experiment time $(\mathrm{t}=3 \mathrm{~min})$ constant (Fig. 3). Frequencies below $3 \mathrm{~Hz}$ led to disordered assemblies. Such frequencies reduced the rate of charging of the objects, because lower rates of collisions between spheres, and lower velocities of rolling on the surface, reduced the rate of tribocharging. In principle, a lower rate of charging should not prevent the formation of ordered assemblies if the time of agitation were extended until the spheres acquire large charges; nevertheless, we found that 
agitating the spheres at frequencies lower than $3 \mathrm{~Hz}$ for one hour did not produce ordered structures, because disordered assemblies could not acquire enough momentum to rearrange into ordered structures. Frequencies of $12 \mathrm{~Hz}$ and higher made the collisions too energetic: collisions of spheres with the walls or other spheres could lift spheres off the surface of the dish (Fig. 3(a)). We note that the spheres can be confined vertically using a lid on top of the dish—as we did in previous studies; ${ }^{21-22}$ for this work, we chose not to use a lid because aluminum (the material of the dish in most of the experiments reported here) is opaque and we did not want to introduce a fourth tribocharging material (a transparent lid) in the system.

Frequencies between 3 and $8 \mathrm{~Hz}$ led to ordered steady-state structures containing hexagonal-layered, rhombic, and square lattice crystallites. As the frequency increased, the crystallites became larger, the proportion of hexagonal-layered domains decreased, and the proportion of square domains increased. The ABN analysis (Fig. 3(c)) showed that as the frequency increased, the assemblies became more ordered overall, and the equilibrium between hexagonal-layered and square structures shifted towards predominantly square structures. The charge on the Nylon and Teflon spheres (Fig. 3(b)) was 1.4 times lower for an agitation at $3 \mathrm{~Hz}\left(\mathrm{Q}_{\mathrm{N}}=465 \pm 75 \mathrm{pC}\right.$ and $\left.\mathrm{Q}_{\mathrm{T}}=-507 \pm 92 \mathrm{pC}\right)$ than for all other frequencies $(4-12 \mathrm{~Hz})$ where the charge was the same $\left(\mathrm{Q}_{\mathrm{N}}=648 \pm 80 \mathrm{pC}\right.$ and $\left.\mathrm{Q}_{\mathrm{T}}=-740 \pm 92 \mathrm{pC}\right)$.

We used frequencies from 6 to $8 \mathrm{~Hz}$ for the rest of the experiments involving spheres, because these frequencies yielded high electrostatic charge and good short- and long-range order during experiments that lasted no more than three minutes, and because the spheres remained in contact with the dish during agitation. 
Fig. 3 Influence of the frequency of agitation on the assembly of Nylon (dark) and Teflon (light) spheres. a) Photographs of the steady-state structures. The assembly made at $12 \mathrm{~Hz}$ has regions with different lattice structures and includes spheres lifted off the surface. b) The average charge on the objects in the steady-state. The error bars represent one standard deviation. c) Computed fractions of spheres in square and hexagonal layered structures (computed fractions are systematically lower than the actual fractions). The error bars are the standard deviation calculated for approximately 500 images from 7 experiments. 

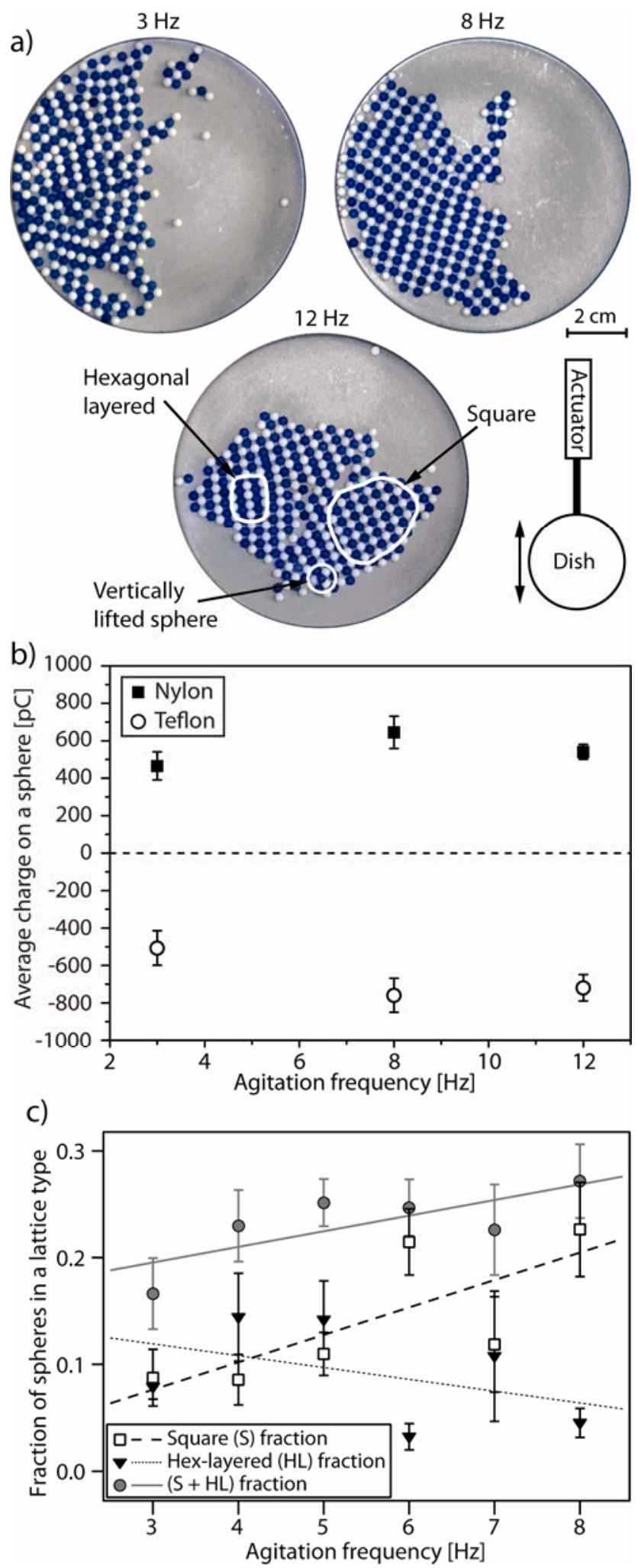
5.1.3. The impact of the shape of the dish. We investigated how the structures formed in aluminum dishes having the same surface area $\left(\mathrm{A}=7854 \mathrm{~mm}^{2}\right)$ but different shapes (Fig. 4); we were in particular interested in comparing triangles and squares (shapes with corners) with circles $(\mathrm{d}=100 \mathrm{~mm})$. A corner confines the spheres, and the local lattices were determined more by this confinement than by the charge on the spheres. In equilateral triangular and square aluminum dishes, Nylon and Teflon spheres $\left(\mathrm{n}_{\mathrm{N}}=\mathrm{n}_{\mathrm{T}}=170, \mathrm{~d}_{\mathrm{N}}=\mathrm{d}_{\mathrm{T}}=\right.$ $3.18 \mathrm{~mm}$ ) formed non-square structures in the corners that were farthest from the linear motion agitator. We did not encounter non-square structures in the corners closest to the linear agitator or perpendicular to the direction of movement. This prevalence of non-square structures forming in the corners away from the agitator was most likely due to a bias in the apparatus: the forward and the return stroke of the linear actuator did not have the same kinematics (velocity and acceleration), therefore the spheres received a larger momentum moving away from the agitator than towards it.

The lattice structure in the corners farthest from the actuator was a mixture of hexagonaldistributed and disordered arrangements of spheres. On triangular dishes, this non-square structure contained up to $35 \%$ of the total spheres and extended about 15 sphere diameters into the dish. On square dishes the non-square structure was smaller, contained up to $25 \%$ of the spheres, and extended about 10 sphere diameters into the dish (Fig. 4). The rest of the spheres on both dishes formed a square lattice in the middle of the dish. This square lattice was the preferred structure, because of the approximately 1:1 ratio of charge between the spheres $\left(\mathrm{Q}_{\mathrm{N}}=600 \pm 50 \mathrm{pC}, \mathrm{Q}_{\mathrm{T}}=-760 \pm 80 \mathrm{pC}\right)$.

In circular dishes, the structure of the assemblies was sometimes distorted near the walls, but these distortions extended at most 2-3 sphere diameters into the dish, and the overall 
Fig. 4 Influence of the shape of the dish on the self-organization of Nylon (blue) and Teflon (white) spheres. The photographs show the steady-state structures of the dishes in various shapes and orientations relative to the linear motion actuator; the schematics indicate the position of the actuator for all images.

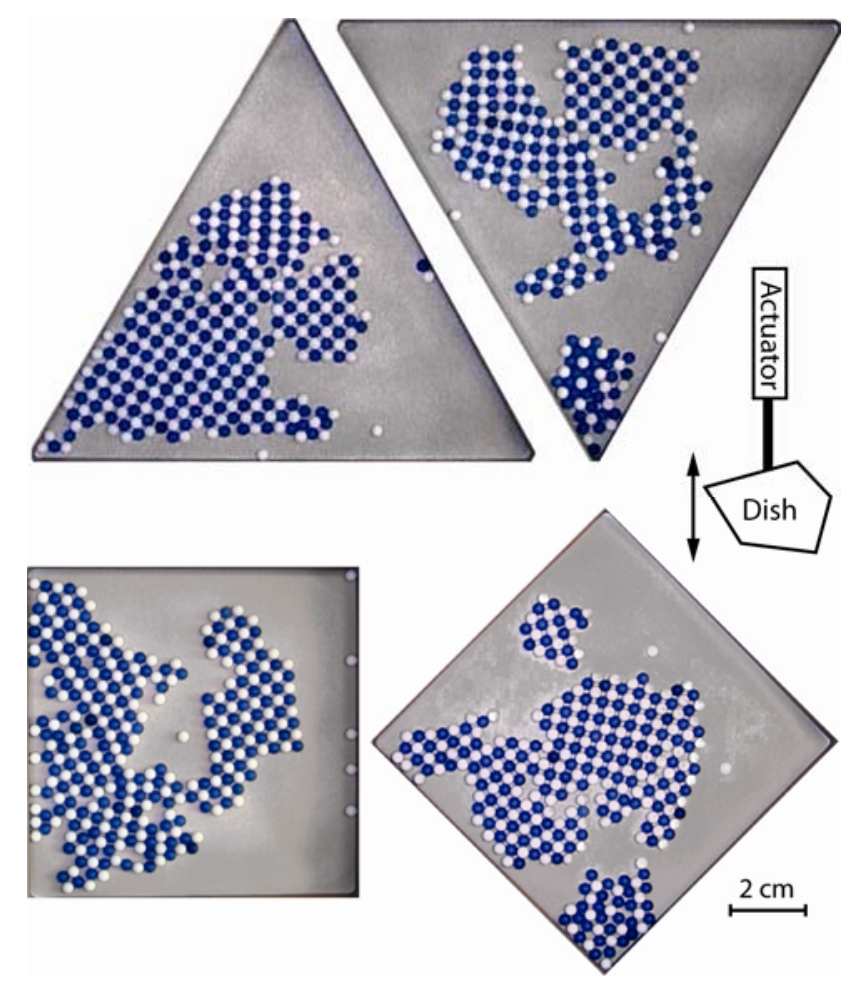


assembly had more uniform lattice structures. We carried out our further experiments using only circular dishes.

5.1.4. The effect of the shape of the objects. We studied the influence of the shape of the objects on self-organization comparing spheres, cubes, and cylinders. We chose spheres, cubes, and cylinders because they are common symmetrical shapes in which a polymeric material can be purchased. Cylinders had the least tendency to form structures with longrange order, especially if their aspect ratio was large; Fig. S7 in the ESI $\uparrow$ shows images of assemblies of cylinders.

The tribocharging and self-assembly of spheres and cubes (Fig. 5) occurs in different ways, because the contact area between the objects during the collisions were different, and because spheres rolled, whereas cubes slid over the dish. While spheres roll and can make contacts over their whole surface, cubes can contact the dish only with their bottom surface and other objects only with their side surfaces. Another distinction between spheres and cubes is that a compact assembly of spheres includes free space, but one of cubes does not. We used Nylon $(\mathrm{N})$ and Teflon $(\mathrm{T})$ spheres and cubes $(\mathrm{d}=3.18 \mathrm{~mm})$ on circular aluminum dishes $(\mathrm{d}=70 \mathrm{~mm})$ in a 1:1 mixture, and we adjusted the amplitude and frequency of the linear agitator to form structures with the best short- and long-range order.

Combinations of spheres and cubes assembled in densely packed arrangements with different degrees of order (Fig. 5(a)). The main difference between cubes and spheres was that cubes moved and tribocharged mostly by sliding against each other and the dish; some cubes occasionally flipped and the rate of flipping in the whole system was on the order of 10 cubes per minute. Inducing the sliding of the cubes required higher dish accelerations than the rolling of the spheres, and we used higher shaking frequencies to induce the self- 
Fig. 5 Influence of the shape of Nylon (dark) and Teflon (light) components on their assembly. a) Photographs of the steady-state structures of 1:1 (left column) and 1:2 (right column) Nylon:Teflon mixtures. The icons indicate the shape and material of the components used (e.g., $\mathrm{N}$ in a circle represents a Nylon sphere). The direction of agitation was vertical. b) The surface charge density, averaged over the whole surface of components, in 1:1 mixtures, after $180 \mathrm{~s}$ of agitation. The error bars represent one standard deviation. 


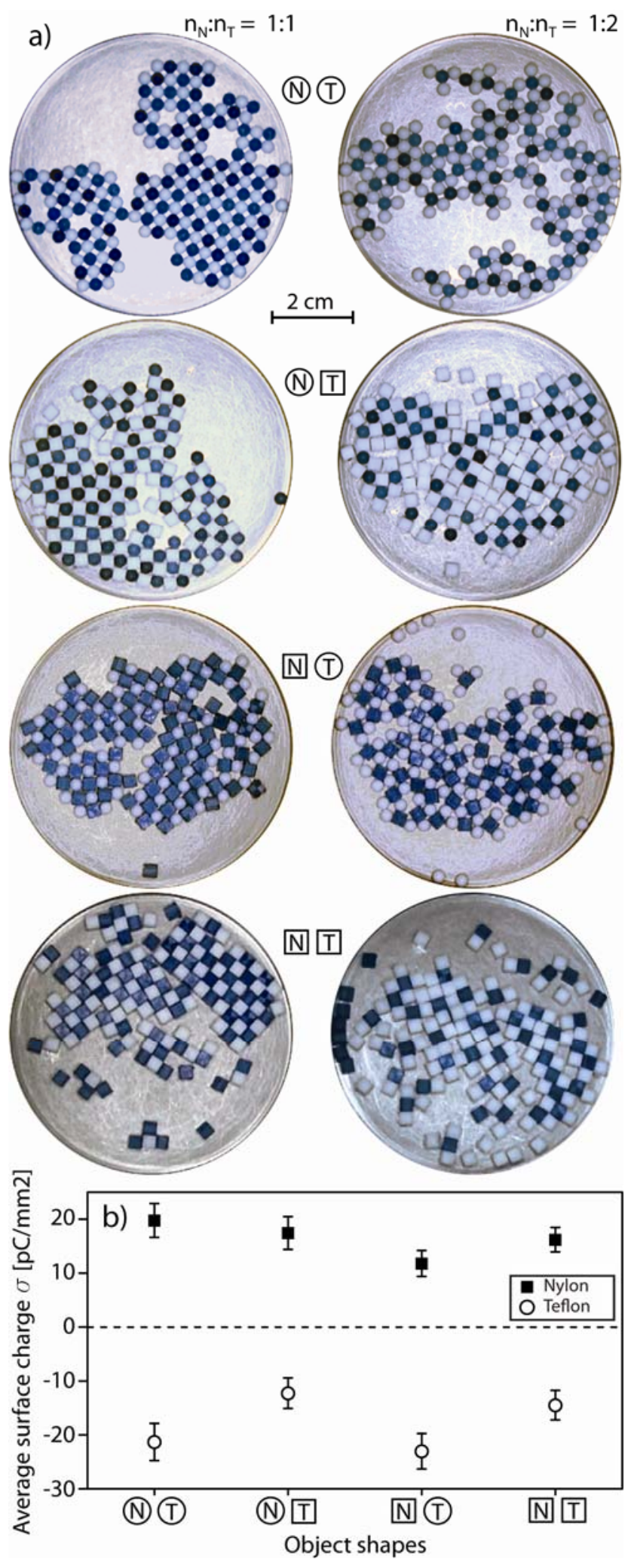


organization of cubes $\left(v_{\text {sphere }}=8 \mathrm{~Hz}, \mathrm{~A}_{\text {sphere }}=8 \mathrm{~mm}, v_{\text {cube }}=14 \mathrm{~Hz}, \mathrm{~A}_{\text {cube }}=10 \mathrm{~mm}, v_{\text {cube+sphere }}\right.$

$\left.=10 \mathrm{~Hz}, \mathrm{~A}_{\text {cube+sphere }}=14 \mathrm{~mm}\right)$.

Cubes tribocharged more slowly, and acquired smaller surface charge densities than spheres after $180 \mathrm{~s}$ of agitation, despite the more energetic agitation; we did not investigate the charging of cubes for durations of agitation longer than three minutes, and it is possible that the charge of these cubes was not fully stabilized. The differences in tribocharging between cubes and spheres were most visible in experiments that used mixtures of cubes and spheres: the surface charge density on cubes was approximately half of the net surface charge density on spheres.

We decided to use only spheres in the systematic studies, because they had the most symmetric shape and their motion (mostly by rolling) was actuated effectively at lower shaking frequencies.

\subsection{Systematic studies of tribocharging in millimeter-scale assemblies}

After determining the best experimental conditions (a linear actuation with $9 \mathrm{~mm}$ amplitude and 6 to $8 \mathrm{~Hz}$ frequency, a circular dish and spherical objects, and a filling ratio of 33\%), we used these conditions to search for different types of self-assembled structures of millimeterscale tribocharged objects, and to understand better the self-assembly process. Our study provided us with guidelines for what conditions to use in order to produce a certain kind of structure, and with several insights about the self-assembly process.

\subsubsection{The effect of the relative diameter of dish and spheres. Dishes of different} diameter have both different surface areas and different wall curvatures. Because we kept the 
diameter of the spheres and the surface coverage constant, a change in the diameter of the dish modified the total number of spheres on the dishes and the size of the spheres relative the curvature of the wall of the dishes - and thus allowed us to vary the degree to which the dish had confining effects on the assembly process (Fig. 6).

We chose hexagonal-distributed structures to study the influence of confinement, because these structures were more affected by confinement than square structures. A hexagonal structure would lose its long-range symmetry when distorted by either impacts with the wall or by changing its shape to match the curvature of the wall, because it is close-packed and any relative movement of the spheres destroys its crystalline symmetry. In contrast, square structures, by shearing into a rhombic structure, can accommodate a certain amount of distortion without losing long-range order.

To study the effect of confinement, we chose Delrin and Torlon spheres $\left(\mathrm{n}_{\text {total }}=54\right.$ to $\left.659, \mathrm{n}_{\text {Delrin }}: \mathrm{n}_{\text {Torlon }}=2: 1, \mathrm{~d}=3.18 \mathrm{~mm}\right)$ on aluminum dishes $(\mathrm{d}=40-140 \mathrm{~mm})$ because they could form highly charged hexagonal structures. In preliminary experiments, this combination of spheres formed the most ordered hexagonal-distributed structures when we used twice as many Delrin as Torlon spheres. The hexagonal-distributed lattice structure was composed of subunits of six Delrin spheres surrounding one Torlon sphere. This mixture of Delrin and Torlon could also from square lattices with a 1:1 ratio of spheres, after expelling excess Delrin spheres from the assemblies.

The ordered hexagonal-distributed lattice structure characteristic of 2:1 Delrin:Torlon mixtures was most affected by confinement in the smallest dish we tested $(\mathrm{d}=40 \mathrm{~mm})$. The hexagonal order was lost and the assembly can be described as either two-dimensional disordered, or as being composed of multiple chains with repeating links made from one 
Fig. 6 Influence of the size of the dish on self-assembly. a) Photographs of the average steady-state structures of Delrin (light) and Torlon (dark) spheres on aluminum dishes of different sizes. The image inserts show typical assembly structures: chains (40-mm dish) and hexagonal-distributed lattices (100-mm dish). b) DBS distribution of assemblies. The dashed vertical lines indicate the expected peak positions of perfect square (S), hexagonal-distributed (HD), and hexagonal-layered (HL) structures (see Fig. S2 in the ESI† for details). 

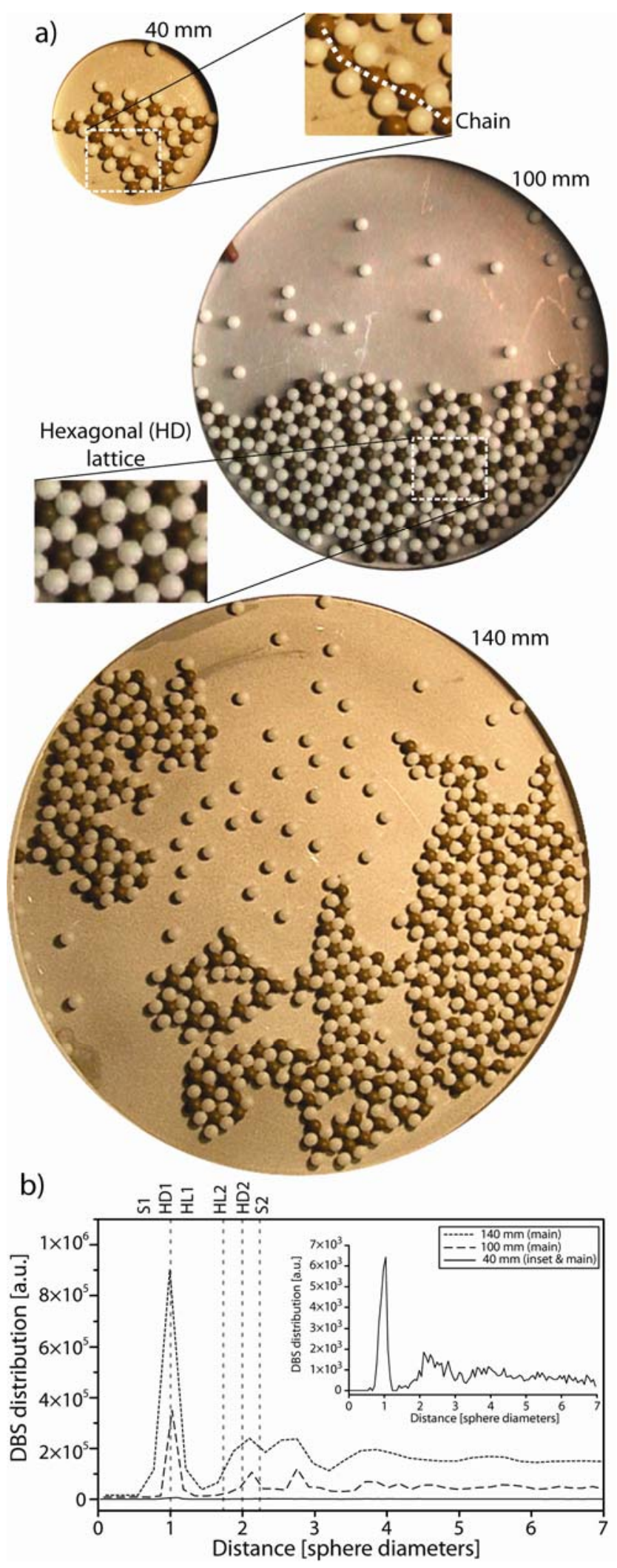
Torlon and two Delrin spheres, as seen in the lower side of the 40-mm dish shown in Fig. $6(\mathrm{a})$.

In dishes with $100 \mathrm{~mm}$ and $140 \mathrm{~mm}$ diameter, the spheres formed ordered, but not perfect, hexagonal-distributed structures (Fig. 6(a)). These structures had a slight excess of Torlon spheres relative to the ideal 2:1 lattice stoichiometry; approximately $20 \%$ of the Delrin spheres were expelled from the close-packed assemblies and spread over the open areas of the dishes. The main differences between assemblies formed in these two dishes were that (i) while a single compact assembly formed in the 100-mm dish, in the larger 140$\mathrm{mm}$ dish the assembly was composed of multiple and loosely-joined crystallites with hexagonal packing (Fig. 6(a)), and (ii) the hexagonal structures formed in the larger dish had more packing defects; the peaks in the DBS distribution were broader than those on the 100mm dish (Fig. 6(b)).

The formation of smaller crystallites on the larger dish than on the mid-sized dishes was due to multiple factors. (i) The early assemblies formed on the larger dish were three to four times larger than the ones formed on the smaller dishes ( 9 to 20 spheres vs. 3 to 5 spheres). This larger size made the assemblies more massive, and reduced the distance they traveled during the time of the experiment. These assemblies moved when momentum was transferred to them through collisions with either the wall or other spheres or assemblies. The larger an assembly was, the larger was the transferred momentum needed to be for it to move. (ii) When two or more of the assemblies came together and formed a larger structure, often a number of unbound Delrin spheres were included inside the larger structures. These unbound Delrin spheres became weak points in the larger structures because they had the same charge. The trapped Delrin spheres repelled one another, and the aggregate around them either 
rearranged and generated an opening, or completely broke apart to release the trapped spheres. We did not see this behavior in the 100-mm dishes, since the number of free Delrin spheres and the size of the first assemblies formed were smaller. (iii) Due to the larger mass of the 140-mm dish, its shaking kinematics was different; we estimated that the peak acceleration was $15 \%$ lower than in the $100-\mathrm{mm}$ dish.

The charge on the spheres was not affected by the size of the dish. Unbound Delrin spheres gained approximately half the charge of bound Delrin spheres, and Torlon spheres gained approximately twice the charge of bound Delrin spheres $\left(\mathrm{Q}_{\text {Torlon }}=-859 \pm 93 \mathrm{pC}\right.$, $\mathrm{Q}_{\text {Delrin }}=498 \pm 112 \mathrm{pC}, \mathrm{Q}_{\text {unbound Delrin }}=297 \pm 92 \mathrm{pC}$ ). The lower charge on unbound Delrin spheres, compared to the bound ones, was due to the fact that unbound Delrin spheres could tribocharge only though contact with the dish; since the dish material was located in the middle of the triboelectric series, unbound Delrin spheres gained less charge than bound Delrin spheres (which tribocharged more because they were in contact with Torlon spheres; Torlon is located at the bottom of the triboelectric series).

We note that the assembly of Delrin and Torlon was electrically neutral. In a first approximation, the assemblies were neutral because the charge on Torlon spheres was approximately twice the charge on Delrin spheres, and both the hexagonal-distributed lattice and the chains formed in the smallest dish had twice the number Delrin spheres as Torlon spheres. At closer inspection, the absolute value of the charge on bounded Delrin was slightly larger than half of the one on Torlon spheres, but when the actual number of bound Delrin spheres (1.6-1.7 times the number of Torlon spheres) was considered, the assemblies were found to be neutral within the error of the measurement. 
5.2.2. The influence of the material of the dish and the spheres. The previous studies on the self-assembly of electrostatically charged spheres ${ }^{20-22}$ chose their materials such that the dish (and some of the spheres), would be located in the middle of the triboelectric series and not charge highly, while the materials of positively- and negatively-charging spheres were chosen from opposite ends of the triboelectric series such that they would acquire charges that were large, equal, but opposite in sign. Such a choice simplifies the system (its behavior can be approximated as being governed by the tribocharging of the two sphere materials) but limits the possibility of discovering a potentially rich set of self-assembly behaviors when all three materials contribute significantly to tribocharging. We therefore investigated the effects of having three different tribocharging materials, following the procedure of varying one of the materials while keeping the other two constant. This procedure allowed us to correlate any changes in the system to the material we changed.

5.2.3. The material of the dish. The material of the dish surface in previous electrostatic self-assembly studies was gold, which is electrically conductive and located in the middle of the triboelectric series. As a result, tribocharging due to contact between spheres and the dish played a minor role on self-assembly. We surveyed several other dish materials, searching for conditions under which tribocharging by contact with the dish played a more important role.

We tested the influence of the material of the dish on the charge and structure of selfassemblies by tribocharging Nylon and Teflon spheres $\left(\mathrm{n}_{\text {total }}=340, \mathrm{~d}=3.18 \mathrm{~mm}\right.$, either separately or as a 1:1 mixture $)$ on circular dishes $(\mathrm{d}=100 \mathrm{~mm})$ made from materials at various positions in the triboelectric series: Nylon, Delrin, aluminum, PMMA and Teflon (Fig. 7). 
Fig. 7 Influence of the chemical composition of the dish on the assembly of Nylon (dark) and Teflon (light) spheres. The photographs show steady-state structures of Nylon and Teflon spheres on dishes with $100 \mathrm{~mm}$ diameter made from various materials. The dish materials were ordered along the vertical axis according their relative position in the triboelectric series, from negative-charging to positive-charging. We modified the color, intensity and contrast of the pictures, and the color of spheres, to increase their visibility. 

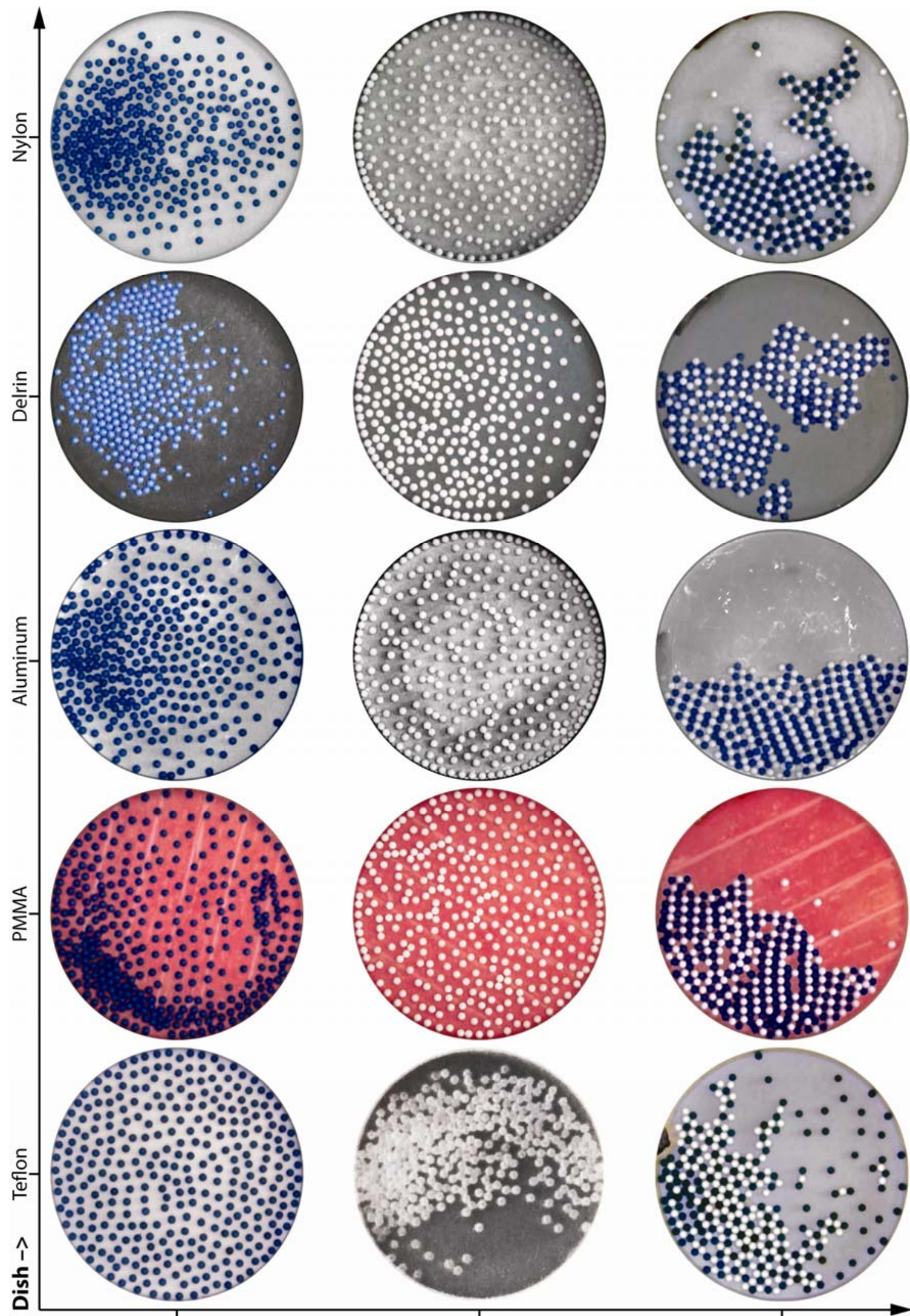

Sphere -> Nylon

Teflon

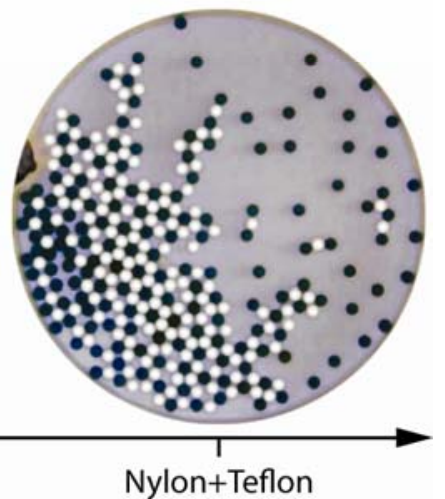


Agitation of spheres of a single type resulted in the tribocharging of spheres in agreement with the relative position in the triboelectric series of the sphere and dish materials. The charge on Nylon spheres changed sign and increased as the position of the material of the dish in the triboelectric series decreased from Nylon to Teflon $\left(\mathrm{Q}_{\mathrm{N}}=-171 \mathrm{pC} \pm 75 \mathrm{pC}\right.$ on Nylon to $\mathrm{Q}_{\mathrm{N}}=606 \mathrm{pC} \pm 122 \mathrm{pC}$ on Teflon, Fig. 8(a)). Teflon spheres, on the other hand, charged similarly on all dishes $\left(\mathrm{Q}_{\mathrm{T}}=-638 \pm 91 \mathrm{pC}\right.$ on Nylon $)$ with the exception of the Teflon dish where the charge was around zero and at our limit of detection. We note that Nylon spheres charged negatively on a Nylon dish; while this behavior was surprising, cases of tribocharging between objects made from the same material have been reported before. ${ }^{37}$ 44,51

The spatial arrangement of single Teflon and Nylon spheres depended on the absolute value of their charge. For absolute charge values below approximately $200 \mathrm{pC}$ (Teflon on Teflon; Nylon on Nylon and Delrin), the spheres were unevenly spread on the surface. As the absolute value of the charge increased, the spheres became distributed more evenly on the surface of the dish, and part of the spheres accumulated at the wall forming a ring with approximately constant spacing between neighboring spheres. Most of the spheres near the wall accumulated there after the agitation stopped, but in some cases part of them assembled at the wall during agitation.

The patterns of single Teflon and Nylon spheres can be explained by their electrostatic interactions. The accumulation of spheres near the wall was due to the electrostatic attraction between the spheres and the wall: because the whole system is electrically neutral, the dish acquired a charge opposite in sign from that of the spheres, and thus the wall of the dish attracted the spheres. The process of accumulation at the wall ceased after a certain number 
Fig. 8 The influence of the material of the dish on Nylon, Teflon, and mixed assemblies. a) Average steady-state charges on the spheres. The polymers on the x-axis were ordered according to their location in the triboelectric series. The distance between the points on the $\mathrm{x}$-axis is arbitrary. b) Computed fractions of spheres in square, hexagonal-layered, and hexagonal-distributed lattices, for mixed assemblies. All error bars represent one standard deviation.
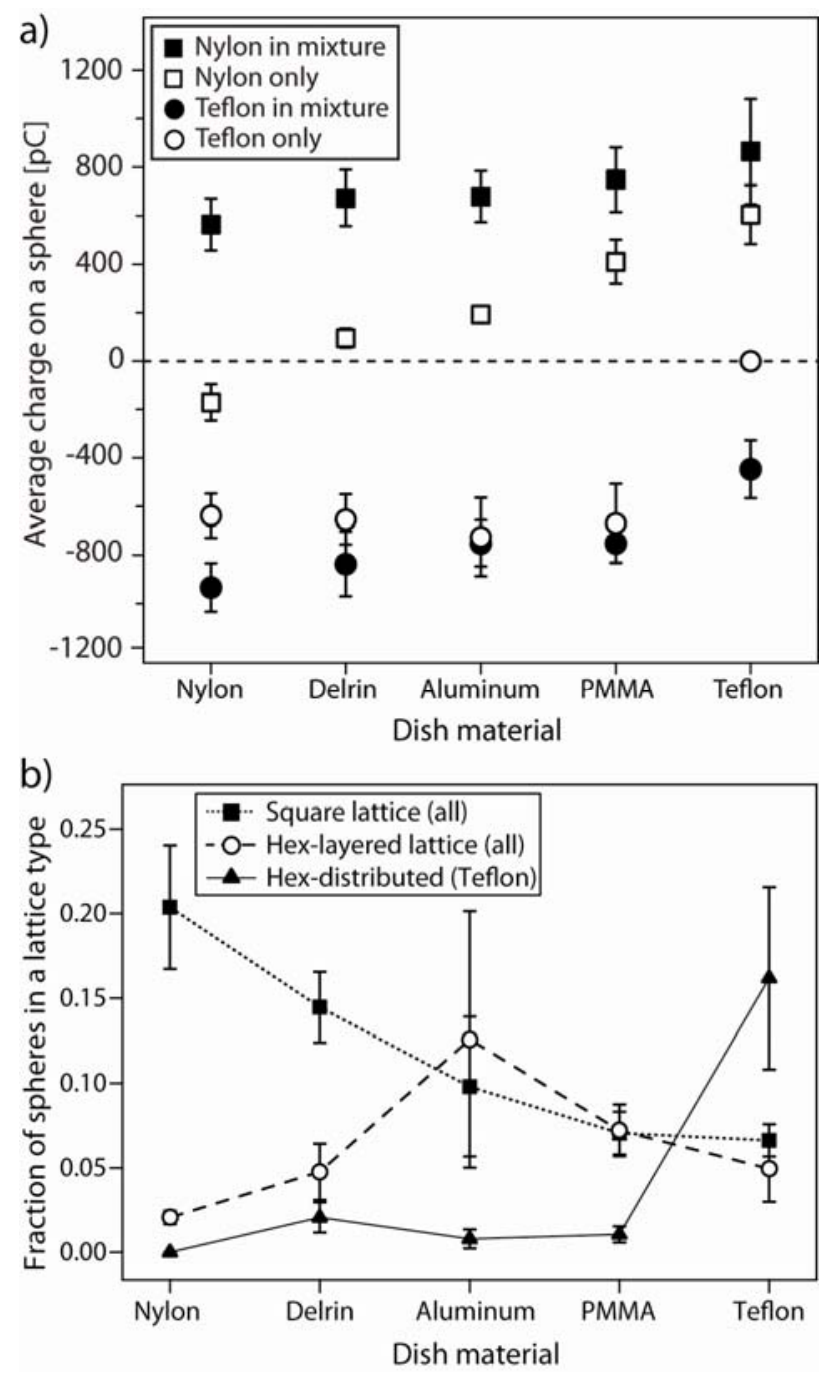
of spheres contacted the wall, because additional spheres, although still attracted by the wall, were repelled more strongly by the accumulated spheres. The spheres which did not come in contact with the wall remained on the surface of the dish, and their distribution was more uniform if they carried more charge and thus experienced stronger repulsive forces from the other spheres.

We often encountered the same type of arrangement (some spheres at the wall, some distributed over the surface) in the cases in which spheres were expelled from mixtures, such as the case of Delrin spheres in experiments with differently sized dishes (Fig. 6(a)). In these cases, the physical explanation was similar, with the exception of the fact that another phenomenon possibly enhanced the accumulation of spheres at the wall: since the dish was made of electrically conductive aluminum, it can attract charged spheres through the electrical polarization of the aluminum, even if the dish is neutral.

The structure of mixed assemblies depended on the material of the dish. The 1:1 mixture of spheres formed (i) loosely-joined crystallites with cubic lattices on Nylon and Delrin dishes, (ii) compact or nearly compact structures with cubic and hexagonal-layered symmetry on aluminum and PMMA dishes, and (iii) a mixture of expelled Nylon spheres, chains, and hexagonal-distributed crystallites on Teflon dishes (Fig. 7).

We determined the fractions of spheres in different types of lattices using the ABN analysis (Fig. 8(b)). The proportion of square assemblies decreased as the position of the material of the dish in the triboelectric series varied form Nylon to Teflon; hexagonal-layered structures were most prevalent in the middle of the series (i.e., on the aluminum dish); and hexagonal-distributed structures (with an excess of Teflon spheres) formed on the Teflon dish only. 
The charge on the spheres also depended on the material of the dish. The charge on Nylon spheres increased 1.5 times (e.g., $\mathrm{Q}_{\mathrm{N}}=565 \pm 107 \mathrm{pC}$ on Nylon and $\mathrm{Q}_{\mathrm{N}}=864 \pm 216$ $\mathrm{pC}$ on Teflon, Fig. 8(a)), and the charge on Teflon decreased two times $\left(\mathrm{Q}_{\mathrm{T}}=-933 \pm 100 \mathrm{pC}\right.$ on Nylon and $\mathrm{Q}_{\mathrm{T}}=-446 \pm 119 \mathrm{pC}$ on Teflon) when the position of the material of the dish in the triboelectric series varied from Nylon to Teflon.

The series of experiments with mixtures on different substrates provided a visual illustration (Fig. 7) of the tendency of the assemblies to be overall electrically neutral. When the dish was made of Delrin, aluminum, and PMMA, the absolute values of the average charges on Nylon and Teflon spheres were approximately equal, and most of the spheres were incorporated in the assemblies. On Nylon and Teflon dishes, the charges were significantly different, and a significant fraction of spheres that carried a larger absolute charge were expelled from the assemblies. On the Teflon dish, the imbalance between the absolute charges of Nylon and Teflon spheres was large enough to lead to the formation of 1:2 (Nylon:Teflon) hexagonal-distributed structures.

5.2.4. The material of the spheres. We mixed Nylon (N) spheres with either polypropylene (PP), high-density polyethylene (HDPE) or polystyrene (PS) spheres in 1:1 mixtures under the same experimental conditions $\left(\mathrm{n}_{\text {total }}=340, \mathrm{~d}=3.18 \mathrm{~mm}, 100-\mathrm{mm}\right.$ circular aluminum dishes) to test the influence of the material of the spheres.

Both the structure and the charge were influenced by the materials involved, and the results fell into two regimes. The spheres assembled into either a mixture of irregular chains and disordered, loosely connected assemblies (N/PP and N/HDPE), or a heterogeneous densely packed assembly that contained hexagonal-layered, rhombic, square lattices and disordered regions (N/PS, Fig. 9). The magnitude of the charge on the loosely packed spheres 
Fig. 9 Dependence of the steady-state structure on the material of the spheres. a) Photographs of the steady-state structures of 1:1 mixtures of Nylon (darker) with HDPE, PP, or PS (lighter) on aluminum dishes. b) DBS distributions of the steady-state structures. c) Average charges on the spheres in the steady-state. The error bars represent one standard deviation. 

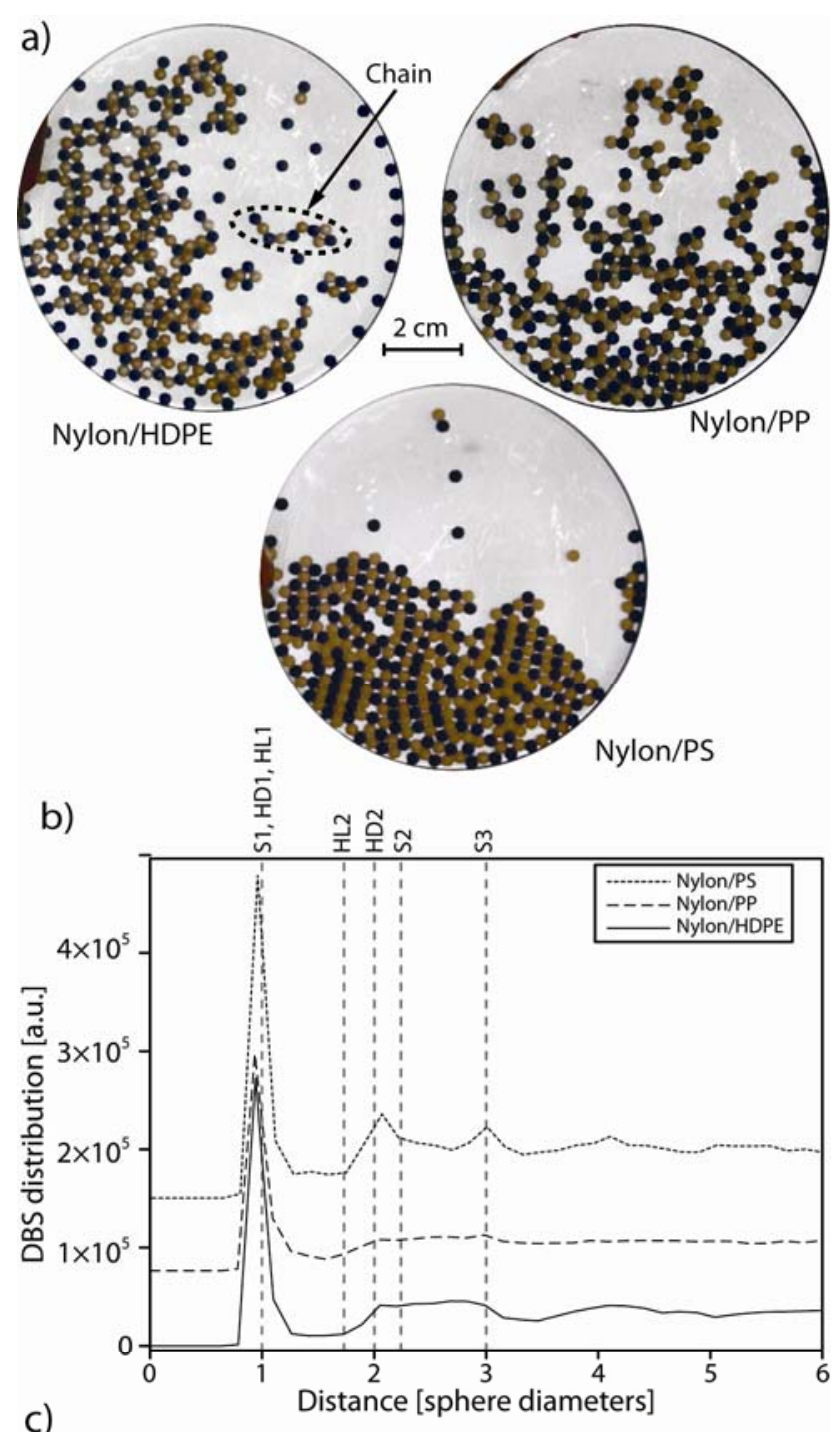

c)

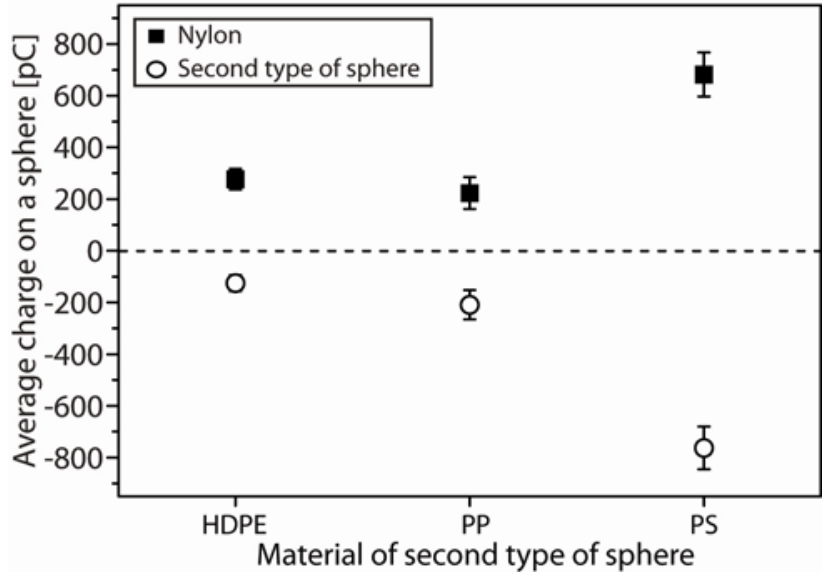


was low $\left(\mathrm{N} / \mathrm{HDPE}: \mathrm{Q}_{\mathrm{N}}=277 \pm 50 \mathrm{pC}, \mathrm{Q}_{\mathrm{HDPE}}=-125 \pm 32 \mathrm{pC} ; \mathrm{N} / \mathrm{PP}: \mathrm{Q}_{\mathrm{N}}=232 \pm 62 \mathrm{pC}, \mathrm{Q}_{\mathrm{PP}}\right.$ $=-209 \pm 57 \mathrm{pC})$ and on densely packed spheres high (N/PS: $\mathrm{Q}_{\mathrm{N}}=682 \pm 86 \mathrm{pC}, \mathrm{Q}_{\mathrm{PS}}=-763 \pm$ $83 \mathrm{pC}$ ). In the case of Nylon and HDPE, Nylon spheres acquired approximately twice the charge of HDPE spheres, and $\sim 20 \%$ of them were expelled from the assemblies. For Nylon/PP and Nylon/PS, the charges on the two types of spheres had approximately the same magnitude and almost all spheres were incorporated in the final assemblies.

The experiments with different types of materials showed that the formation of closepacked assemblies requires relatively large charges $(|\mathrm{Q}|>500 \mathrm{pC}$ on $3.18 \mathrm{~mm}$ spheres). Spheres that developed smaller steady-state charges $(|\mathrm{Q}| \sim 200 \mathrm{pC})$ tended to form chains (one-dimensional structures) rather than compact two-dimensional assemblies.

5.2.5. The effect of the relative diameter of two types of spheres. We were motivated to study the assembly of spheres with different sizes by the possible analogy with ionic crystals formed of ions of different sizes: a change in the size of the ions can change the lattice structure in simple ionic crystals; crystals of $\mathrm{NaCl}$, which are composed of ions of approximately the same size, have a face-centered cubic structure, while crystals of $\mathrm{CsCl}$, composed of ions of different sizes, have a primitive cubic structure. We mixed Nylon and Teflon spheres with different diameters $(\mathrm{d}=2.38 \mathrm{~mm}$ to $\mathrm{d}=6.35 \mathrm{~mm}$; the Nylon spheres were always smaller) on circular dishes from 80 to $140 \mathrm{~mm}$ in diameter, while keeping the filling fraction of the dish constant at 33\% (Fig. 10).

As the ratio of diameters of Nylon and Teflon spheres increased from 0.37 to 1 , we observed a transition from chain-type structures to square or hexagonal-layered lattice structures (Fig. 10(a)). The proportion of spheres that formed irregular chains generally decreased as the ratio of diameters approached unity, but the simultaneous trend of 
Fig. 10 Influence of the relative size of Nylon (dark) and Teflon (light) spheres on their selfassembly. a) Photographs of steady-state structures. b) Ratio of absolute charges on the two types of spheres as a function of the ratio of their surface areas. c) Ratio of absolute surface charge densities on the two types of spheres. All error bars represent one standard deviation of charge measurements. 

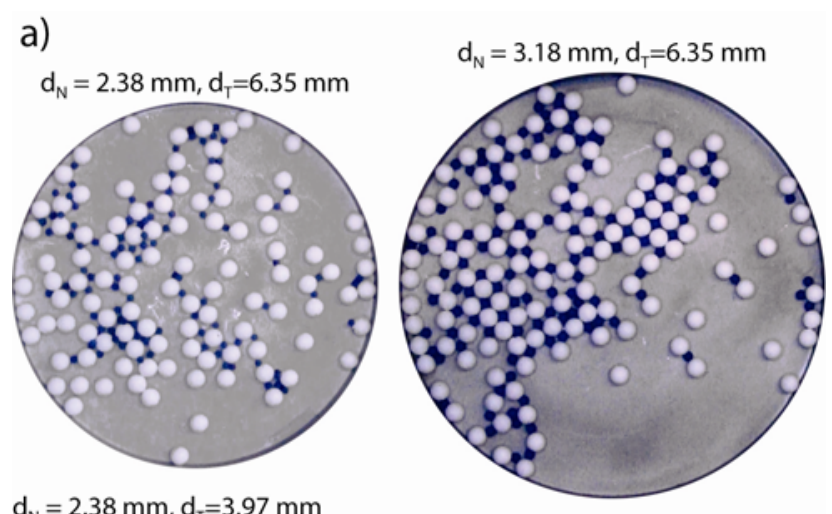

$\mathrm{d}_{\mathrm{N}}=2.38 \mathrm{~mm}, \mathrm{~d}_{\mathrm{T}}=3.97 \mathrm{~mm}$

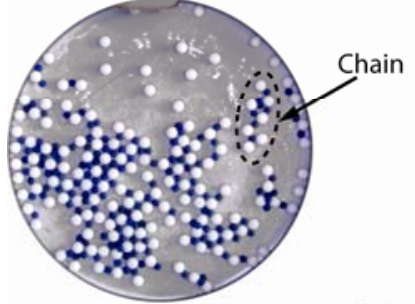

$\mathrm{d}_{\mathrm{N}}=2.38 \mathrm{~mm}, \mathrm{~d}_{\mathrm{T}}=3.18 \mathrm{~mm}$
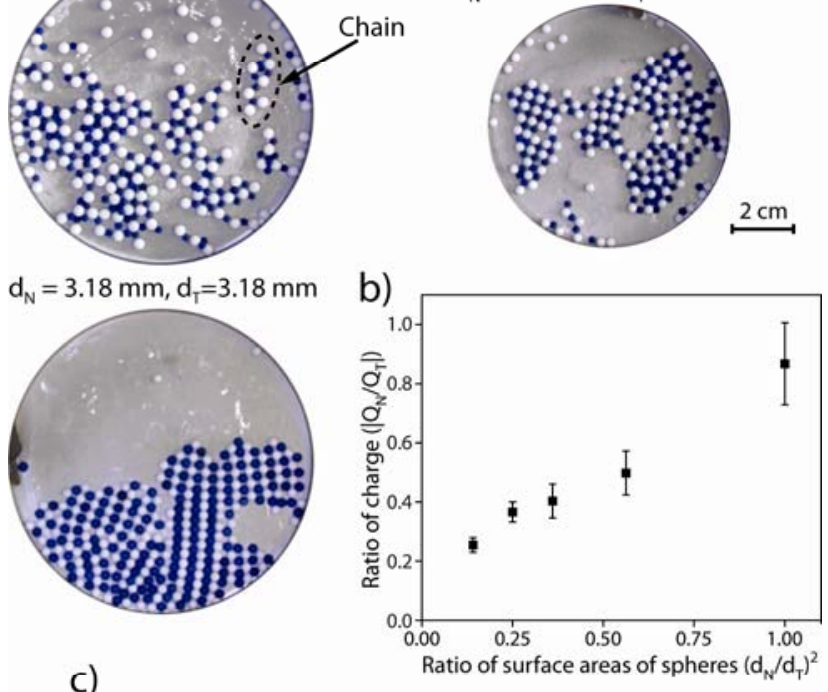

c)

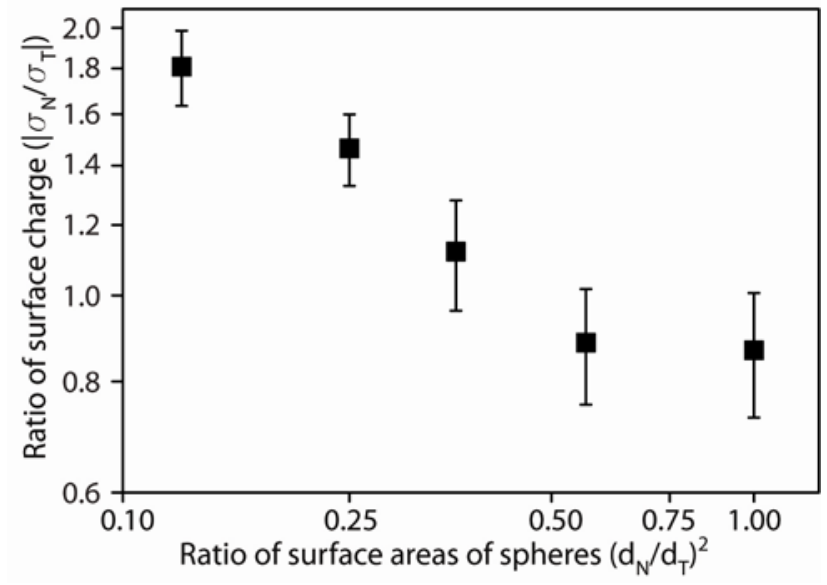


of forming more closely packed assemblies was not monotonic, probably due to geometric or steric effects. For a ratio of sphere diameters of 0.37 , the close-packed assemblies were disordered; for 0.5 , square; for 0.6 , hexagonal; for 0.75 , square; and for 1 , square and hexagonal-layered. When the sphere diameters were different, some small spheres adhered to the large ones and were lifted off the dish.

The ratio of charges and surface charge densities followed monotonic and opposite trends. Nylon spheres carried more charge (Fig. 10(b)) but less surface charge density (Fig. 10(c)) relative to the Teflon spheres as their diameter (relative to the Teflon spheres) increased. If the sphere diameters were different, Teflon spheres carried more charge and a significant fraction of Teflon spheres were expelled from the assembly.

The results of experiments with different sized spheres had two unusual features: the formation of chain structures, and the imbalance of charges and surface charge densities. The ease with which chains formed for the mixtures with spheres with different diameters can be understood as an electrostatic phenomenon. The larger spheres had larger absolute charges, therefore the chains had net charge; after assembly, the chains repelled each other.

5.2.6. The influence of the concentration of two types of spheres. We reported that both the total concentration of spheres on a square dish and the number ratio of spheres influenced the structures formed. ${ }^{20,22}$ In these experiments, using equal numbers of Nylon and Teflon spheres always led to square assemblies independent of the their total concentration, and pentagonal and hexagonal structures formed when the numbers were different. In our previous work, we assumed that a sphere of a given type acquires the same charge independent of the number and type of contacts, and attributed the formation of nonneutral pentagonal or hexagonal structures to electrical polarization. 
We showed here that a sphere of a given material acquires different steady-state charges when it is in contact with different dish materials, or with spheres from a range of different materials. Therefore, at least in our self-assembly system, the steady-state charge on a sphere of a given material is not simply a material property, but depends on the other materials in the system. It is plausible, furthermore, that even if all materials are kept the same, the steady-state charge depends on the relative concentration of the spheres. A single Nylon sphere immersed in Teflon spheres will have more tribocharging contacts than the same sphere in a 1:1 mixture of Teflon and Nylon spheres; therefore at least its rate of charging will depend on the ratio of Nylon and Teflon spheres. If the steady-state charge also depends on this ratio, it will be possible to form, depending on the ratio of spheres, a range of different structures that are neutrally charged.

We investigated the possible effect of sphere ratio on the assembly structure and on the sphere charges by mixing a constant total number of Nylon and Teflon spheres $\left(n_{\text {total }}=339, d\right.$ $=3.18 \mathrm{~mm}$, circular aluminum dish $\mathrm{d}=100 \mathrm{~mm}$ ) while varying the number fraction of Nylon spheres $\chi_{N}\left(\chi_{N}=n_{N} /\left(n_{N}+n_{T}\right)\right)$ from 0.1 to 0.9 (Fig. 11, Fig. S8 in the ESI $\dagger$ ).

Both the lattice structure and the charge on the spheres depended on the number fraction of Nylon spheres, and the assemblies that formed were always approximately neutral electrically. A given type of sphere acquired more charge when its proportion decreased, but the charge was less than directly proportional to the rate of contacts with other type of sphere (Fig. 11(b), Fig. S8(a) in the ESI $\dagger$ ). If the sphere was of the less abundant type, its charge was usually larger than that of the other type of spheres.

For $\chi_{\mathrm{N}}=0.5$, the magnitude of the charge on both Nylon and Teflon spheres was similar $\left(\mathrm{Q}_{\mathrm{N}}=677 \pm 98 \mathrm{pC}\right.$ and $\left.\mathrm{Q}_{\mathrm{T}}=-780 \pm 98 \mathrm{pC}\right)$, and most spheres were incorporated into a 
Fig. 11 Influence of the relative concentration of Nylon (dark) and Teflon (light) spheres on their assembly. A) Photographs of the steady-state structures. b) Computed fractions of spheres in 1:1 lattices (S and HL) and 2:1 (HD) types of lattices. c) Average charge of the spheres. All error bars represent one standard deviation. 

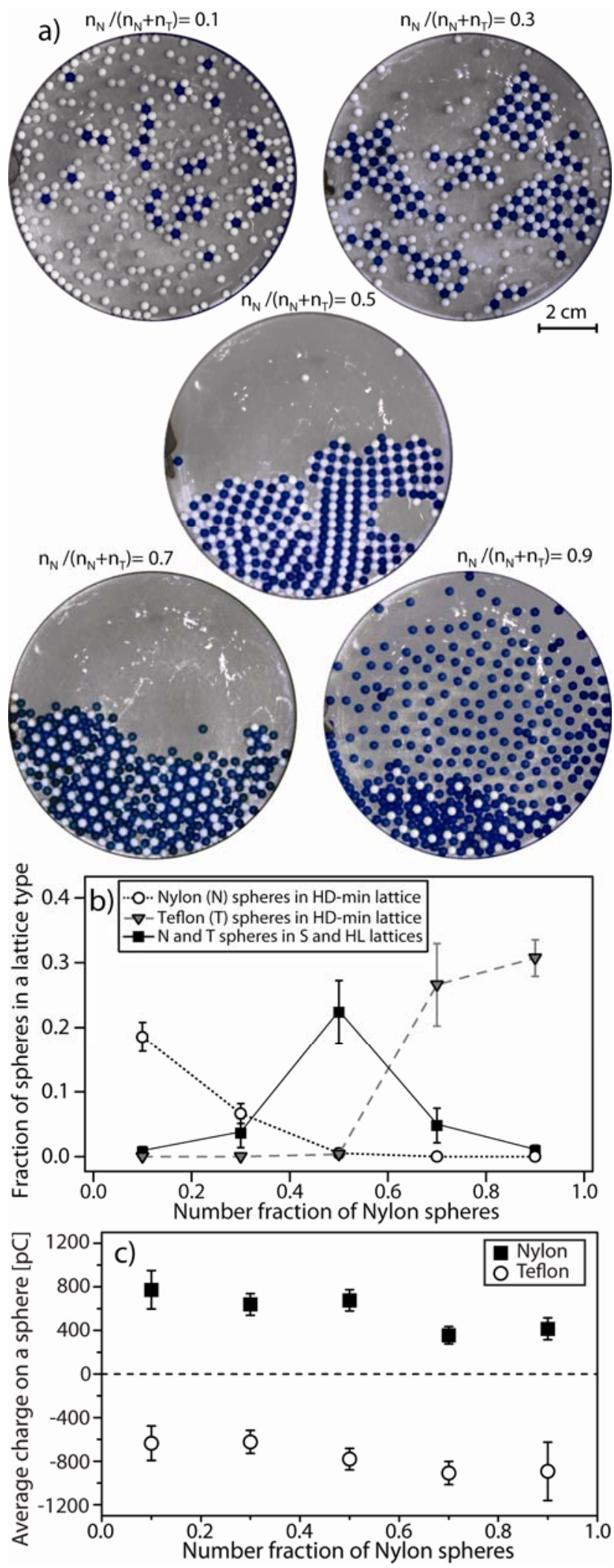
structure that had approximately equal numbers of spheres in square and in hexagonallayered lattices (Fig. 11(a)), and was electrically neutral.

For $\chi_{N}=0.7$ the charge on Teflon was approximately 2.6 times that of the Nylon spheres $\left(\chi_{\mathrm{N}}=0.7: \mathrm{Q}_{\mathrm{N}}=355 \pm 80 \mathrm{pC}\right.$ and $\left.\mathrm{Q}_{\mathrm{T}}=-909 \pm 106 \mathrm{pC}\right)$. Most spheres were incorporated into a hexagonal-distributed lattice structure with twice as many Nylon as Teflon spheres (Fig. 11(a)), and each Teflon sphere surrounded by six Nylon spheres. The reversed sphere composition, $\chi_{\mathrm{N}}=0.3$, was characterized by almost equal charges on Nylon and Teflon $\left(\chi_{\mathrm{N}}=\right.$ 0.3: $\mathrm{Q}_{\mathrm{N}}=639 \pm 100 \mathrm{pC}$ and $\mathrm{Q}_{\mathrm{T}}=-623 \pm 105 \mathrm{pC}$ ), and led to loosely packed disordered assemblies in which the less abundant Nylon spheres were surrounded by four or five Teflon spheres (Fig. 11(a)).

Experiments performed at the largest imbalance between numbers of Nylon and Teflon spheres, $\chi_{N}=0.1$ and $\chi_{N}=0.9$, led to the formation of 'rosettes' composed of one of the less abundant type of sphere surrounded by four to six spheres of the other type (Fig. 11(a)); some 'rosettes' aggregated together but did not assemble into structures with long-range order. For $\chi_{N}=0.1$, Nylon-center 'rosettes' incorporated four or five Teflon spheres, were scattered across the dish, and often shared their Teflon spheres forming disordered chains. For $\chi_{\mathrm{N}}=0.9$, Teflon-center 'rosettes' incorporated five or six Nylon spheres and were clustered together, often sharing their Nylon spheres and forming a disordered close-packed aggregate.

For both $\chi_{\mathrm{N}}=0.1$ and $\chi_{\mathrm{N}}=0.9$ experiments, many of the spheres present in larger fraction were not part of the assemblies and were scattered across the dish or stuck near the wall. Despite the similarity in the structures formed, these two 'symmetric' experiments led 
to different charge ratios between the less and the more abundant type of sphere $\left(\chi_{\mathrm{N}}=0.1\right.$ :

$\mathrm{Q}_{\mathrm{N}}=773 \pm 177 \mathrm{pC}, \mathrm{Q}_{\mathrm{T}}=-635 \pm 158 \mathrm{pC}$ and $\mathrm{Q}_{\mathrm{N}} / \mathrm{Q}_{\mathrm{T}}=1.2 \pm 0.4 ; \chi_{\mathrm{N}}=0.9: \mathrm{Q}_{\mathrm{N}}=415 \pm 100$ $\mathrm{pC}, \mathrm{Q}_{\mathrm{T}}=-893 \pm 270 \mathrm{pC}$ and $\mathrm{Q}_{\mathrm{N}} / \mathrm{Q}_{\mathrm{T}}=0.5 \pm 0.2$ ). Nylon spheres had 1.2 times the absolute charge of Teflon spheres at $\chi_{\mathrm{N}}=0.1$, and Teflon spheres had 2.2 times the absolute charge of Nylon spheres at $\chi_{\mathrm{N}}=0.9$.

The electrical neutrality of self-assembled crystals with different sphere ratios is illustrated by the combination of the ABN analysis (Fig. 11(b)) and charge measurements (Fig. 11(c)). The structures with a 1:1 sphere ratio (square and hexagonal-layered) are dominant when the number of Nylon and Teflon spheres is equal and their net charges are approximately equal. If either Nylon or Teflon spheres are present in a larger number, the less abundant sphere accumulates more charge and tends to be the minority component in $2: 1$ hexagonal-distributed assemblies.

\section{Discussion}

\subsection{The electrical neutrality of the crystalline lattice.}

The system that we reported here is an evolution of the system we developed previously to examine the two-dimensional electrostatic self-assembly of millimeter-sized polymer objects. ${ }^{20}$ Many, but not all, of our observations confirmed those reported in this previous study. In particular, $\mathrm{w}^{20}$ reported that some of the crystalline assemblies were not electrically neutral - that these crystals had a substantial net charge. In the experiments 
described here, we found that all steady-state close-packed assemblies were approximately electrically neutral. $^{52}$

To elucidate this significant discrepancy between the two studies, we verified that our present results were not dependent on the material of the dish (see Fig. S9 in the ESI $\dagger$ ). Mixtures of Torlon and Delrin spheres in gold-coated dishes (used in our previous studies), and in aluminum dishes, charged similarly and the steady-state assemblies did not have substantial net charge.

We conclude that our previous conclusion that lattices can have substantial net charge was erroneous. We arrived at this conclusion by numerical modeling based on the assumption that lattices of objects that were present in different proportions still had the same charges (but opposite in sign) — an assumption that does not correspond to the physical properties of our system. This assumption was based on the observation that Nylon and Teflon spheres charge to the same values of opposite sign; we have shown here that this charging behavior occurred only when the number of Nylon and Teflon spheres was equal. When Nylon and Teflon spheres are mixed in different proportions, they acquire charges of different magnitudes.

\subsection{Tribocharging in a three-material system}

To our knowledge, tribocharging in a three-material system was not studied systematically as a scientific phenomenon to date - a fact that is probably due to the complexity of the system: even the simpler case of tribocharging in a two-material system is a complex and not fully understood process. Tribocharging in a three-material system has nevertheless technological 
importance, playing a key role the separation of recyclable plastics by tribocharging and electrostatic separation. ${ }^{53-54}$ Here we started to investigate such a system by testing the hypothesis that the charge acquired by the objects is a materials property, and by asking what are new or less studied processes are involved in three-material tribocharging.

One of the simplest hypotheses one can make about tribocharging is that the charge (or the density of surface charge) acquired by the contacting objects depends on the materials of the objects only. Both in this work and previously we observed that after a sufficiently long duration of agitation, the charges of spheres reached steady-state values. The steady-state charge of a sphere made from a given material depended on at least these experimental parameters: the material of the dish, the type and frequency of agitation, the material of the other type of sphere, and the number ratio of the two types of spheres. Even when all materials and the kinetics of agitation were the same, spheres of different diameters acquired different charges and different surface charge densities (Fig. 10). In our multi-component, three-material tribocharging system, the steady-state charge and the steady-state density of surface charge are not material properties.

The triboelectric series offers a qualitative guide for understating the behavior of a threematerial system. In waste separation applications, the best separations were achieved when the material of the tribocharging container was located, within the triboelectric series, approximately halfway between the materials to be separated; ${ }^{54}$ in our system, we observed that the most ordered structures formed under similar conditions (Fig. 7). Waste separation and the self-assembly of spheres occurred nevertheless for other arrangements of the three materials within the series as well, facts which illustrate the limitations of rationalizing the system based on the triboelectric series alone. 
A distinctive characteristic of tribocharging in a system containing three or more materials is the competition between several types of contact electrification (e.g. material 1 with material $2 ; 1$ with 3 ; etc.). This competition leads to processes that were not investigated before, such as the redistribution of charge between two objects that might carry more charge than the steady-state charge they would acquire just by contact with each other. In our experiments, such a situation might have occurred when a Nylon sphere came in contact with the aluminum dish after it charged by contact with Teflon spheres. Because the separation in the triboelectric series between Nylon and Teflon is larger than the separation between Nylon and aluminum, we expect the Nylon spheres to charge more when in contact with the Teflon spheres, and thus be 'overcharged' during a subsequent contact with the aluminum dish.

\subsection{The kinetics of the self-assembly process}

We observed, in agreement with our previous work,${ }^{20}$ that under linear agitation the formation of ordered 2D lattices from initially uncharged spheres has in three stages: (i) most of the spheres from aggregates made from small numbers of spheres, (ii) the small aggregates from a larger, partially disordered aggregate, and (iii) the larger aggregate rearranges ('anneals') into a crystalline structure.

This assembly process is not analogous to nucleation and growth (the most common way in which ionic and molecular crystals form) because: (i) the initial aggregates (two-five spheres) are too small to have a well-defined lattice structure and therefore cannot be regarded as classical crystallization nuclei (which have the same structure as the stable phase) and (ii) most of the spheres become part of these initial aggregates. It might be 
possible to find conditions under which the self-assembly occurs in a way analogous to nucleation; we believe that such conditions should include spheres that charge lightly (such that the driving force for self-assembly becomes smaller) and the introduction of a 'nucleus' made from spheres with steady-state charges arranged in the final crystal structure. ${ }^{55}$ Another method for reducing the tendency of charged spheres to assemble quickly could be to add a third type of non-charging spheres to 'dilute' the system. In our experiments, we found the closest analogy to the nucleation and growth process during experiments in which we used orbital agitation (Fig. S5 in the ESI $\uparrow$ ); orbital agitation is more gentle then linear agitation. A possible microscopic analogy of the self-assembly process under linear agitation is crystallization at very large degrees of supersaturation; the mechanism of such crystallization is not well understood but it is an intermediate between nucleation and spinodal decomposition. ${ }^{56-58}$ Another possible analogy is with the processes of nanoparticle formation and aggregation. ${ }^{59-61}$

\subsection{Formation of self-assembled chains}

We found that the self-organization of electrically charged objects can in some cases lead to the formation of chains rather than to compact two-dimensional assemblies. The structure of these chains ranges from the ordered 2:1 Delrin:Torlon chains (Fig. 6(a)) to disordered and interlocked chains (Fig. 9(a), Fig 10(a)); disordered and interlocked chains can be difficult to identify in static pictures such as the ones we presented here, but their presence is obvious if one watches the system under agitation. The conditions under which we sometimes observed chain formation were: (i) confinement by a small dish (Fig. 6(a)); (ii) low steady-state 
charges (Fig. 9(a)); (iii) different sphere diameters (Fig. 10(a)); and (iv) different numbers of similarly-charging spheres (Fig. 11(a)). These conditions suggest that the organization of spheres in chains occurs when either the forces that drive self-assembly are small (weak electrostatic charges), or when the formation of a lattice is frustrated by electrostatic repulsion between chains, dish confinement, steric constraints (spheres with very different diameters cannot pack easily), or scarcity of one type of spheres. The richness of chain morphologies, and of experimental conditions under which they appear, suggests that chains are a common result of electrostatic self-assembly.

Whether chain structures form in ionic systems (such as salts precipitating in a solvent) is not currently known, due to the difficulty of observing such structures at the atomic scale. The self-assembly of chain structures has been observed in nanoparticle systems ${ }^{62}$ and seems to be driven (in the absence of templates, or of particle functionalization) by electric ${ }^{63}$ or magnetic ${ }^{64}$ dipole-dipole interactions; further self-assembly of nanoparticle chains is prevented by electrostatic repulsion between chains. ${ }^{62}$

\subsection{The relationship between charge and structure during the self-assembly of tribocharged objects}

The defining characteristic of our system, when compared to other electrostatic selfassembled structures, is the relationship between the electrical charge of the objects and the lattice structures they form. This relationship is caused by (i) the continuous range of values of charge that the objects can acquire and by (ii) the dependency of tribocharging of one object on the characteristics of the system (the neighbors' material and size, and the 
frequency of contacts with its neighbors). The relation between charge and structure is more important than in ionic systems (where structure influences the electrical polarization but not the charge), and also dynamic: the environment (spatial structure and distribution of charge) that an object experiences evolves during self-assembly. This relationship largely determines the self-assembly process (proceeding from clusters to aggregates to crystalline aggregates) and also the 'pattern-switching' phenomena ${ }^{20}$ in which lattices transition between different structures and compositions.

The relationship between charge and structure was too complex to quantify. We found, however, that a main rule governing it is that self-assembled close-packed structures must be electrically neutral, and this rule allowed us to explain why mixtures of Nylon and Teflon spheres in different proportions (Fig. 11) form different lattice structures (when the fractions of spheres are equal, 1:1 lattices form, and when they are imbalanced, 2:1 lattices form). If one of the types of spheres is in minority, it acquires more charge because it is more likely to make tribocharging contacts with the other type of spheres. To form electrically neutral close-packed structures, the minority sphere must be the less abundant type in a structure with different fractions of sphere types, such as a hexagonal-distributed lattice.

\section{Conclusions}

We have investigated the electrostatic self-assembly of millimeter-sized objects, using a system that we developed previously ${ }^{20}$ to demonstrate electrostatic self-assembly in mixtures of two types of polymer spheres. Compared to our previous work, ${ }^{20}$ we improved the experimental setup and procedures, we surveyed systematically the effect of most of the 
experimental parameters on self-assembly, and we discovered new features of the selfassembly. The goal of our work was to develop a setup that would be appropriate for the investigation of a wide range of self-assembly phenomena, and to identify directions for further studies.

We were able to form close-packed crystalline structures under many distinct experimental conditions: self-assembly is robust. Two other types of structures-'rosettes' and chains - also formed frequently, indicating that at least three classes of structures (crystals, chains, and 'rosettes') can form during self-assembly. Each class of structure was favored by certain characteristics of the system: crystals formed when the objects have large charges and are agitated with sufficient energy; chains formed when the formation of the crystal is frustrated by electrostatic repulsion or steric factors; and 'rosettes' were characteristic of mixtures in which there is a large imbalance in the fractions of the two types of spheres

In our previous study ${ }^{20}$ we reported that some of the self-assembled lattice structures could have substantial net charge — an unexpected result which we failed to replicate in our present study. After investigating a wide range of system parameters (the materials and sizes of the sphere and the dish materials and sizes, and the characteristics of agitation), we conclude that the close-packed structures formed by self-assembly, despite their different compositions and lattice structures, are always approximately electrically neutral.

While the build-up of charge on the spheres and the dish during agitation is a part of the self-assembly process, it also represents an interesting contact electrification problem: tribocharging in a three-material system. There are relatively few studies of this problem, despite the fact that the process is used industrially for the separation of recyclable plastics. 
We found that the triboelectric series is a useful concept for choosing a set of sphere and dish materials that are more likely to form sphere lattices, and for understanding qualitatively the assembly of spheres, but we could not make quantitative predictions of electrification based on the triboelectric series. We are investigating the possibility of using our system to produce quantitative tribocharging data and new insights into the tribocharging in systems composed of more than two types of materials.

The electrical charge acquired by the objects was not a characteristic of the material of the objects but depended on many experimental parameters, including the structure of the lattice. The relationship between the charge of the objects and their lattice structure during assembly is one of the most important characteristics of self-assembly in our system. The charge-structure interaction distinguishes our system from other electrostatic self-assembled structures, both material (such as ionic crystals) and more exotic (such as Coulomb crystals), and underlines the necessity of performing measurements of charge of the objects in the ordered lattice in addition to determining the structure of the lattice.

The dependence of the charge of the objects on the experimental conditions limits the relevance of our system as a model for ionic systems, but if this dependence can be understood and controlled, it also has the potential to make this type of self-assembly more versatile than ionic crystallization. We are particularly interested in continuing the work presented here to study phase transitions, rates of charging and self-assembly, and tribocharging in systems containing more than two types of materials. We believe that the component-level understanding of electrostatic self-assembly of millimeter-sized components—-particularly when these model systems are designed to mimic microscopic 
phenomena such as phase transitions - will lead to the discovery of new phenomena and of useful analogies with the behavior of microscopic matter.

\section{Acknowledgments}

We thank Dr. Siowling Soh for information about electrostatic charge measurements. This work was supported by the US Department of Energy, Division of Materials Sciences \& Engineering, under Award No. DE-FG02-OOER45852. Vivian M. Tran and Daryl Vulis, respectively, acknowledge the 2011 and 2010 Harvard REU programs funded by the National Science Foundation under grants no. DMR-1005022 and DMR-0649199.

\section{Notes and references}

$\dagger \quad$ Electronic Supplementary Information (ESI) available: (1) a selection of materials (including the ones we used in our experiments) ranked in a triboelectric series; (2) a description of the experimental apparatus; (3) a detailed description of the image analysis procedures; (4) additional data and images describing the effect of dyeing the objects, the stability of charge, the assembly process under different types of agitation, mechanisms of structural rearrangements, the self-assembly of Teflon and Nylon spheres mixed in different proportions, and the effect of using gold instead of aluminum dishes; and (5) a list of the movie files that are included in the supplementary information.. See DOI: 10.1039/b000000x/ 
1. J. W. Mullin, Crystallization, 4 edn., Butterworth-Heinemann, Oxford, UK and Woburn, MA, 2001.

2. I. Sunagawa, Crystals: Growth, Morphology, and Perfection, 1 edn., Cambridge University Press, 2007.

3. W. A. Tiller, The Science of Crystallization: Microscopic Interfacial Phenomena, 1 edn., Cambridge University Press, 1991.

4. A. Van Hook, Crystallization: Theory and Practice, 1 edn., Reinhold Publishing Co., New York, 1961.

5. O. Pouliquen, M. Nicolas and P. D. Weidman, Phys. Rev. Lett., 1997, 79, 3640-3643.

6. G. D. Scott, Nature, 1960, 188, 908-909.

7. L. Bragg and J. F. Nye, Proc. Roy. Soc. Lond. A, 1947, 190, 474-481.

8. H. Thomas, G. E. Morfill, V. Demmel, J. Goree, B. Feuerbacher and D. Mohlmann, Phys. Rev. Lett., 1994, 73, 652-655.

9. M. Drewsen, C. Brodersen, L. Hornekaer, J. S. Hangst and J. P. Schiffer, Phys. Rev. Lett., 1998, 81, 2878-2881.

10. J. R. Abo-Shaeer, C. Raman, J. M. Vogels and W. Ketterle, Science, 2001, 292, 476-479.

11. P. Schall, I. Cohen, D. A. Weitz and F. Spaepen, Science, 2004, 305, 1944-1948.

12. H. Yoshida, J. Yamanaka, T. Koga, N. Ise and T. Hashimoto, Langmuir, 1999, 15, 26842702.

13. V. J. Anderson and H. N. W. Lekkerkerker, Nature, 2002, 416, 811-815.

14. P. de Moor, T. P. M. Beelen, B. U. Komanschek, O. Diat and R. A. van Santen, J. Phys. Chem. B, 1997, 101, 11077-11086. 
15. U. Gasser, E. R. Weeks, A. Schofield, P. N. Pusey and D. A. Weitz, Science, 2001, 292, 258-262.

16. P. N. Pusey and W. Vanmegen, Nature, 1986, 320, 340-342.

17. A. vanBlaaderen, R. Ruel and P. Wiltzius, Nature, 1997, 385, 321-324.

18. E. R. Weeks, J. C. Crocker, A. C. Levitt, A. Schofield and D. A. Weitz, Science, 2000, 287, 627-631.

19. B. A. Grzybowski and G. M. Whitesides, J. Phys. Chem. B, 2001, 105, 8770-8775.

20. B. A. Grzybowski, A. Winkleman, J. A. Wiles, Y. Brumer and G. M. Whitesides, Nat. Mater., 2003, 2, 241-245.

21. G. K. Kaufman, M. Reches, S. W. Thomas, J. Feng, B. F. Shaw and G. M. Whitesides, Appl. Phys. Lett., 2009, 94, 044102.

22. G. K. Kaufman, S. W. Thomas, M. Reches, B. F. Shaw, J. Feng and G. M. Whitesides, Soft Matter, 2009, 5, 1188-1191.

23. M. Reches, P. W. Snyder and G. M. Whitesides, P. Natl. Acad. Sci. USA, 2009, 106, 17644-17649.

24. R. R. Dogonadze, The Chemical Physics of Solvation, Elsevier, Amsterdam; New York, 1988.

25. B. J. Kim, J. Tersoff, S. Kodambaka, M. C. Reuter, E. A. Stach and F. M. Ross, Science, 2008, 322, 1070-1073.

26. B. S. Lee, G. W. Burr, R. M. Shelby, S. Raoux, C. T. Rettner, S. N. Bogle, K. Darmawikarta, S. G. Bishop and J. R. Abelson, Science, 2009, 326, 980-984.

27. M. Matsumoto, S. Saito and I. Ohmine, Nature, 2002, 416, 409-413.

28. Reviakine, W. Bergsma Schutter and A. Brisson, J. Struct. Biol., 1998, 121, 356-362. 
29. H. M. Zheng, R. K. Smith, Y. W. Jun, C. Kisielowski, U. Dahmen and A. P. Alivisatos, Science, 2009, 324, 1309-1312.

30. P. E. Hillner, A. J. Gratz, S. Manne and P. K. Hansma, Geology, 1992, 20, 359-362.

31. T. A. Land, J. J. DeYoreo and J. D. Lee, Surf. Sci., 1997, 384, 136-155.

32. J. V. Macpherson, P. R. Unwin, A. C. Hillier and A. J. Bard, J. Am. Chem. Soc., 1996, 118, 6445-6452.

33. A. J. Malkin, Y. G. Kuznetsov and A. McPherson, J. Cryst. Growth, 1999, 196, 471-488.

34. J. C. Wilcke, Disputatio physica experimentalis de electricitatibus contrariis, Rostock, 1757.

35. A. F. Diaz and R. M. Felix-Navarro, J. Electrostat., 2004, 62, 277-290.

36. P. E. Shaw and C. S. Jex, Proc. Roy. Soc. Lond. A, 1928, 118, 108-113.

37. J. Lowell and A. C. Rose-Innes, Adv. Phys., 1980, 29, 947-1023.

38. L. S. McCarty and G. M. Whitesides, Angew. Chem. Int. Edit., 2008, 47, 2188-2207.

39. D. J. Lacks and R. M. Sankaran, J. Phys. D Appl. Phys., 2011, 44, 453001.

40. S. Piperno, H. Cohen, T. Bendikov, M. Lahav and I. Lubomirsky, Phys. Chem. Chem. Phys., 2012, 14, 5551-5557.

41. S. Friedle and S. W. Thomas, Angew. Chem. Int. Edit., 2010, 49, 7968-7971.

42. S. W. Thomas, S. J. Vella, M. D. Dickey, G. K. Kaufman and G. M. Whitesides, J. Am. Chem. Soc., 2009, 131, 8746-8747.

43. Y. P. Chang, Y. C. Chiou and R. T. Lee, Wear, 2004, 257, 347-358.

44. H. T. Baytekin, B. Baytekin, S. Soh and B. A. Grzybowski, Angew. Chem. Int. Edit., 2011, 50, 6766-6770. 
45. S. W. Thomas, S. L. Vella, G. K. Kaufman and G. M. Whitesides, Angew. Chem. Int. Edit., 2008, 47, 6654-6656.

46. S. J. Vella, X. Chen, S. W. Thomas, X. H. Zhao, Z. G. Suo and G. M. Whitesides, J. Phys. Chem. C, 2010, 114, 20885-20895.

47. C. G. Camara, J. V. Escobar, J. R. Hird and S. J. Putterman, Nature, 2008, 455, 10891092.

48. T. Pahtz, H. J. Herrmann and T. Shinbrot, Nat. Phys., 2010, 6, 364-368.

49. M. M. Apodaca, P. J. Wesson, K. J. M. Bishop, M. A. Ratner and B. A. Grzybowski, Angew. Chem. Int. Edit., 2010, 49, 946-949.

50. Dr. Siowling Soh, private communication. Precharged Nylon and Teflon spheres incresed their charge by at most $2-4 \%$ after 20 contacts with PBT twezzers.

51. H. T. Baytekin, A. Z. Patashinski, M. Branicki, B. Baytekin, S. Soh and B. A. Grzybowski, Science, 2011, 333, 308-312.

52. The only assembly that did have an overall charge was made from Nylon and HDPE spheres (Fig. 9). This assembly was not close packed and did not exhibit long-range order.

53. I. Inculet, G. S. P. Castle and J. D. Brown, Particul. Sci. Technol., 1998, 16, 91-100.

54. A. Iuga, L. Calin, V. Neamtu, A. Mihalcioiu and L. Dascalescu, J. Electrostat., 2005, 63, 937-942.

55. We note that simply immobilizing a few spheres together (e.g. by using an adhesive) does not produce a crystallization nucleus because the spheres cannot tribocharge as mobile spheres do.

56. K. Binder, C. Billotet and P. Mirold, Z. Phys. B Cond. Mat., 1978, 30, 183-195. 
57. L. S. Bartell and D. T. Wu, J. Chem. Phys., 2007, 127, 174507.

58. L. R. Gomez and D. A. Vega, Phys. Rev. E, 2011, 83, 021501.

59. V. Privman, D. V. Goia, J. Park and E. Matijevic, J. Colloid Interf. Sci., 1999, 213, 3645.

60. H. Colfen and S. Mann, Angew. Chem. Int. Edit., 2003, 42, 2350-2365.

61. F. C. Meldrum and H. Colfen, Chem. Rev., 2008, 108, 4332-4432.

62. Z. Y. Tang and N. A. Kotov, Adv. Mater., 2005, 17, 951-962.

63. J. H. Liao, K. J. Chen, L. N. Xu, C. W. Ge, J. Wang, L. Huang and N. Gu, Appl.Phys. AMater., 2003, 76, 541-543.

64. H. Wang, Q. W. Chen, L. X. Sun, H. P. Qi, X. Yang, S. Zhou and J. Xiong, Langmuir, 2009, 25, 7135-7139. 